\title{
Coalescence of Euclidean geodesics on the Poisson-Delaunay triangulation
}

\author{
DAVID COUPIER ${ }^{1}$ and CHRISTIAN HIRSCH${ }^{2}$ \\ ${ }^{1}$ Laboratoire Paul Painlevé U.M.R. CNRS 8524, Université Lille 1, France. \\ E-mail: david.coupier@math.univ-lille1.fr \\ ${ }^{2}$ Mathematisches Institut, Ludwig-Maximilians-Universität München, Theresienstraße 39, 80333 München, \\ Germany.E-mail:hirsch@math.lmu.de
}

Let us consider Euclidean first-passage percolation on the Poisson-Delaunay triangulation. We prove almost sure coalescence of any two semi-infinite geodesics with the same asymptotic direction. The proof is based on an argument of Burton-Keane type and makes use of the concentration property for shortest-path lengths in the considered graphs. Moreover, by considering the specific example of the relative neighborhood graph, we illustrate that our approach extends to further well-known graphs in computational geometry. As an application, we show that the expected number of semi-infinite geodesics starting at a given vertex and leaving a disk of a certain radius grows at most sublinearly in the radius.

Keywords: Burton-Keane argument; coalescence; Delaunay triangulation; first-passage percolation; Poisson point process; relative neighborhood graph; sublinearity

\section{Introduction and main results}

\subsection{Introduction}

In the seminal papers $[15,16]$, Licea and Newman have shown coalescence of a large class of geodesics with the same direction in the standard two-dimensional lattice first-passage percolation (FPP) model. To be more precise, for Lebesgue-almost every direction, any two geodesics with this direction starting from different initial points coalesce with probability 1 .

It is expected that coalescence of geodesics with the same direction should be a property that holds not only for $\mathbb{Z}^{2}$ but for a large class of two-dimensional FPP models. However, as of today, this fundamental feature has only been verified for two isolated model extensions. On a planar homogeneous Poisson point process Howard and Newman consider a FPP model with edge weights given by a power of the Euclidean distance [11,12]. On the Poisson-Delaunay triangulation Pimentel considers a FPP model with independent and identically distributed weights on the edges. Although vertices in that model are distributed at random, the independence of edge weights turns out to be sufficiently powerful to transfer local modification arguments from the lattice to the Delaunay triangulation.

In the present paper, we develop a technique that opens up a novel possibility to prove coalescence of geodesics for models where modifying single edge weights is not possible. In our approach, a central role is played by a modified FPP model, where geodesics are forbidden to 
backtrack behind certain vertical lines. For streamlining the presentation, we focus on a Euclidean FPP model on the Delaunay triangulation, where the edge weights are dependent and given by the Euclidean length $v_{1}(e)$ of the corresponding edge $e$. Due to the strong dependence between edge weights and the underlying graph structure, the local modification arguments do not carry over to the present setting. Moreover, we show that our general approach is not restricted to the Delaunay triangulation, but can also be applied to other graphs of interest in computational geometry. As a specific example, we provide explicitly the adaptations that are needed to deal with the Poisson relative neighborhood graph. In fact, the approach of non-backtracking FPP has the potential to be of use in more general FPP models, as long as a concentration of path lengths can be established.

In order to state our main results precisely, we first recall the definitions of the Delaunay triangulation and the relative neighborhood graph and refer the reader to [13] for further properties. Let us start with a set of vertices in $\mathbb{R}^{2}$ given by a homogeneous Poisson point process $X$ whose intensity is assumed to be equal to 1 . Then, the Delaunay triangulation $\operatorname{Del}(X)$ on $X$, is the geometric graph on the vertex set $X$, where an edge is drawn between two vertices $x, y \in X$ if and only if there exists a disk whose intersection with the vertex set $X$ consists precisely of the points $x$ and $y$. The relative neighborhood graph $\operatorname{Rng}(X)$ on $X$, is the geometric graph on the vertex set $X$ where an edge is drawn between two vertices $x, y \in X$ if and only if there does not exist a vertex $z \in X$ such that $\max \{|x-z|,|y-z|\}<|x-y|$. In particular, almost surely, the relative neighborhood graph is a subgraph of the Delaunay triangulation. In the following, many results hold for both $\operatorname{Del}(X)$ and $\operatorname{Rng}(X)$. Hence, we write $G(X)$ as notation to represent either of these graphs. Also, letters $x, x^{\prime}, y, z$ will refer to elements of $X$.

Next, let us introduce a Euclidean FPP model on $G(X)$ and explain the notion of geodesics. Let $P, P^{\prime} \in \mathbb{R}^{2}$ be points that are contained on the edge set of $G(X)$. Then, we denote by $\ell\left(P, P^{\prime}\right)=$ $\ell_{G(X)}\left(P, P^{\prime}\right)$ the Euclidean length $\nu_{1}(\gamma)$ of the shortest Euclidean path $\gamma$ on $G(X)$ connecting $P$ and $P^{\prime}$. That is,

$$
\ell\left(P, P^{\prime}\right)=\inf \left\{v_{1}(\gamma): \gamma \text { is a path on } G(X) \text { connecting } P \text { and } P^{\prime}\right\} .
$$

For any path $\gamma$ on $G(X)$ and $P, P^{\prime} \in \gamma$, we write $\gamma\left[P, P^{\prime}\right]$ for the subpath of $\gamma$ starting at $P$ and ending at $P^{\prime}$. If the path $\gamma$ satisfies $\ell\left(P, P^{\prime}\right)=v_{1}\left(\gamma\left[P, P^{\prime}\right]\right)$ for all $P, P^{\prime} \in \gamma$, then $\gamma$ is called geodesic.

The present paper investigates geodesics $\gamma$ on $G(X)$ that are semi-infinite in the sense that $\gamma$ emanates from a certain starting point but consists of infinitely many vertices. Moreover, writing $S^{1}=\left\{P \in \mathbb{R}^{2}:|P|=1\right\}$ for the unit circle, we say that a semi-infinite path $\gamma$ on $G(X)$ admits an asymptotic direction $\hat{u} \in S^{1}$ if and only if

$$
\lim _{\substack{|y| \rightarrow \infty \\ y \in \gamma}} \frac{y}{|y|}=\hat{u} .
$$

Let $\hat{u} \in S^{1}$ be an arbitrary direction. Based on the classical arguments developed in $[11,15]$, it is proved in [10] that almost surely, for every point $x \in X$, there exists a unique semi-infinite geodesic starting at $x$ and with asymptotic direction $\hat{u}$. Using the terminology of [15], this 
geodesic is called $\hat{u}$-unigeodesic and will be denoted by $\gamma_{x}$ in the following. Note that $\gamma_{x}$ obviously depends on the direction $\hat{u}$, but since in our paper this direction will always be clear from the context, we adhere to the simplified notation.

\subsection{Coalescence of geodesics}

The first main result of our paper establishes the coalescence of $\hat{u}$-unigeodesics.

Theorem 1. Consider Euclidean FPP on $G(X)$. Then, for any given direction $\hat{u} \in S^{1}$, with probability 1 any two geodesics with asymptotic direction $\hat{u}$ eventually coalesce. That is, $\gamma_{x} \cap$ $\gamma_{x^{\prime}} \neq \varnothing$ for all $x, x^{\prime} \in X$.

On a very general level, the proof of Theorem 1 is based on the Burton-Keane technique that has emerged as a powerful tool in the analysis of random planar trees $[6,11,15,17]$. However, the implementation of the Burton-Keane argument for Euclidean FPP on the Poisson-Delaunay triangulation $\operatorname{Del}(X)$ and the Poisson relative neighborhood graph $\operatorname{Rng}(X)$ is markedly different from the examples that have been discussed in the literature so far. Let us explain why.

The classical argument of Burton-Keane starts by assuming that there exist $\hat{u}$-unigeodesics that do not coalesce. Then, a local modification argument is used to show that each of these geodesics has a positive probability to be surrounded by a protective shield. This shield prevents that distant geodesics coalesce with it. In particular, the expected number of shielded - and therefore non-coalescent - geodesics in a bounded box grows as the box area. This contradicts the fact that the expected number of edges of $G(X)$ crossing the box boundary grows as the box perimeter, that is, as the square root of the box area. In a given FPP model, the difficulty of carrying out this program lies in the local modification step.

For instance, in the setting of i.i.d. edge weights [15,17], a direct modification of edge weights makes it possible to generate shields that are avoided by the geodesics starting outside such obstacles. Indeed, by choosing sufficiently large weights, these geodesics are forced to circumvent the shielded area. Conversely, in Euclidean FPP models such as the ones considered in [11] and the present paper, the weights are determined by the locations of the vertices given by a Poisson point process. There is no additional source of randomness on which one could rely. Hence, any local modification step must modify the Poisson point process itself. The classical Euclidean FPP model considered in [11] is based on the complete graph on a Poisson point process where weights are given by certain powers of the Euclidean distance. In particular, the absence of a graph topology entails a powerful monotonicity property: removing Poisson points can only increase shortest-path lengths. This makes it possible to create obstacles by deleting Poisson points in a large region without modifying the geodesics.

The main challenge in analyzing Euclidean FPP on the Delaunay triangulation $\operatorname{Del}(X)$ and the relative neighborhood graph $\mathrm{Rng}(X)$ lies in the lack of a related monotonicity property. Indeed, in both models, the removal of Poisson points has two opposite effects. First, this invalidates paths passing through a deleted vertex, and then increases shortest-path lengths (as in the previous Euclidean FPP models). Second, deleting vertices also has the possibility of unblocking certain edges which can potentially decrease shortest-path lengths when they appear. See Figure 1. We 


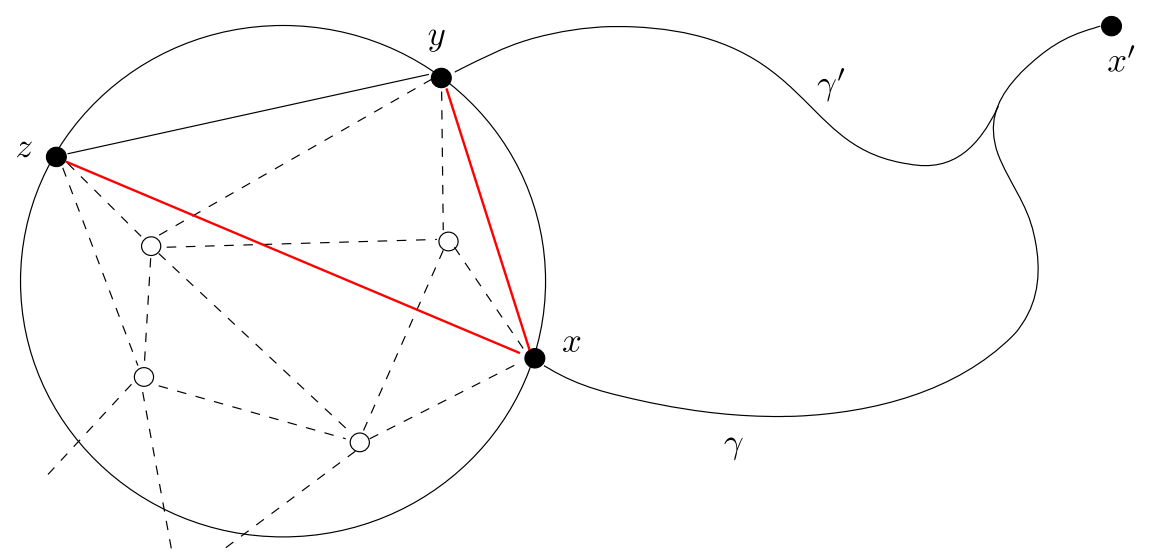

Figure 1. Here is a naive picture explaining the self-healing property of the Delaunay triangulation. Let $x, y, z, x^{\prime}$ be some points of the PPP $X$ and $\gamma$ be the geodesic between $x$ and $x^{\prime}$ w.r.t. $G(X)=\operatorname{Del}(X)$. Let $\bar{X}$ be the process $X$ without its vertices in the interior of the circumcircle defined by $x, y, z$ : the deleted vertices are the white points. The passage from $\operatorname{Del}(X)$ to $\operatorname{Del}(\bar{X})$ removes the edges involving the white points (the dotted lines) and unblocks new edges which are $\{x, y\}$ and $\{x, z\}$. The one connecting $y$ and $z$ was already in $\operatorname{Del}(X)$. Although the geodesic $\gamma$ did not use the removal vertices, it is possible that the geodesic between $x$ and $x^{\prime}$ w.r.t. $\operatorname{Del}(\bar{X})$, say $\gamma^{\prime}$, prefer to pass through a new edge and then will be different from $\gamma$. In this picture, $\gamma^{\prime}$ passes through $\{x, y\}$ before reaching $x^{\prime}$.

refer to this characteristic as self-healing property. This property makes it much more difficult to capture the effects of removing points. Therefore, Euclidean FPP on the Delaunay triangulation $\operatorname{Del}(X)$ and the relative neighborhood graph $\operatorname{Rng}(X)$ are markedly different from the FPP models previously considered in the literature $[11,15,17]$. This requires us to give to the Burton-Keane approach of $[11,15]$ a new twist.

The key idea for the proof of Theorem 1 is to consider a modified Euclidean FPP model in which geodesics are preserved under certain local modifications. More precisely, this modified FPP model forbids geodesics to backtrack behind a given vertical line. This allows us to implement local modifications to the left of that line without influencing geodesics to the right of it. Hence, we are able to construct in the modified FPP model a family of non-coalescent geodesics whose mean number grows as the box area. However, in general these new geodesics are no longer geodesics in the original FPP model.

In order to illustrate the generality of our approach, we stress that shortest-path lengths on the Delaunay triangulation may behave quite differently to shortest-path lengths on the relative neighborhood graph. Indeed, it is known that the Delaunay triangulation is a spanner [8, 14]. That is, when constructing the Delaunay triangulation from an arbitrary locally finite set, any two vertices can be connected by a path whose length is at most $4 \sqrt{3} \pi / 9$ times the Euclidean distance between these vertices. In contrast, starting from an arbitrary infinite set, the relative neighborhood even need not necessarily be connected. Nevertheless, using a Poisson point process as vertex set, Euclidean FPP both on the Delaunay triangulation and the relative neighborhood graph are well-behaved asymptotically. More precisely, as a key tool in the proof 
of Theorem 1, we leverage recently established results on concentration of shortest-path lengths on the Poisson-Delaunay triangulation and the Poisson relative neighborhood graph [10]. Even if the spanner-property of the Delaunay triangulation makes it impossible to punish the passage through obstacles by arbitrarily high costs, the strong concentration of shortest-path lengths implies that creating obstacles with moderately high costs is sufficient for achieving the desired shielding effect.

\subsection{Sublinearity of the number of geodesics leaving large disks}

Let $x^{\star}$ be the closest Poisson point to the origin $o \in \mathbb{R}^{2}$. We denote by $\mathcal{T}_{x^{\star}}$ the collection of geodesics $\gamma_{x^{\star}, x}$, for any $x \in X$, where $\gamma_{x^{\star}, x}$ is the shortest Euclidean path on $G(X)$ connecting $x^{\star}$ and $x$. The continuous nature of the underlying Poisson point process $X$ ensures the a.s. uniqueness of geodesics. Hence, $\mathcal{T}_{x^{\star}}$ is a.s. a tree rooted at $x^{\star}$, called the shortest-path tree on the graph $G(X)$ w.r.t. the $\operatorname{root} x^{\star}$.

A natural question on the shortest-path tree $\mathcal{T}_{x^{\star}}$ concerns the number of its infinite geodesics. To our knowledge, this question was first formulated in the seminal paper of Hammersley and Welsh [9] as the highways and byways problem. Let $\chi_{r}$ be the number of semi-infinite geodesics in $\mathcal{T}_{x^{\star}}$ crossing the circle $S_{r}(o)=r S^{1}$. In Section 8 of [9], the authors wonder if (a) $\chi_{r} \rightarrow \infty$ almost surely as $r \rightarrow \infty$ and (b) if so, how fast. The answer to the first question is yes and the way to state it is now well understood. Combining the moderate deviations result obtained in [10], Theorem 2, for the geodesics $\gamma_{x, x^{\prime}}$ (w.r.t. the straight line segment connecting $x$ and $x^{\prime}$ ) and the general method developed in [12], Proposition 2.8, the following statement holds with probability 1: for every direction $\hat{u} \in S^{1}$, there exists a semi-infinite geodesic in $\mathcal{T}_{x^{\star}}$ starting from $x^{\star}$ with asymptotic direction $\hat{u}$.

Besides, to our knowledge, the question (b) remained unanswered until very recently and the general method developed by one of the authors, initially in [2], then in [5]. This method provides a partial reply to (b): the expectation of $\chi_{r}$ is asymptotically sublinear. See Theorem 2 below.

A semi-infinite geodesic of $\mathcal{T}_{x^{\star}}$ crossing various times $S_{r}(o)$ must be counted only once by $\chi_{r}$. Hence, let us consider the graph obtained from $\mathcal{T}_{x^{\star}}$ after deleting any geodesic $\left(x^{\star}, x_{2}, \ldots, x_{n}\right)$ with $n \geq 2$ (except the endpoint $x_{n}$ ) such that the Poisson points $x^{\star}, x_{2}, \ldots, x_{n-1}$ belong to the disk $B_{r}(o)=\left\{P \in \mathbb{R}^{2}:|P| \leq r\right\}$ but not $x_{n}$. Then, $\chi_{r}$ counts the unbounded connected components of this graph. Thus, let us consider a direction $\hat{u} \in S^{1}$ and a real number $c>0$. Among the previous unbounded connected components, the ones coming from an edge $\left(x_{n-1}, x_{n}\right)$ whose segment $\left[x_{n-1} ; x_{n}\right]=\left\{\lambda x_{n-1}+(1-\lambda) x_{n}: \lambda \in[0,1]\right\}$ crosses the arc of the circle $S_{r}(o)$ centered at $r \hat{u}$ and with length $c$ are counted by $\chi_{r}(\hat{u}, c)$. Using this notation, we state the following theorem.

Theorem 2. Let $\hat{u} \in S^{1}$ and $c>0$. Then,

$$
\lim _{r \rightarrow \infty} r^{-1} \mathbb{E} \chi_{r}=0 \quad \text { and } \quad \lim _{r \rightarrow \infty} \mathbb{E} \chi_{r}(\hat{u}, c)=0 .
$$

The first limit of (2) can be understood as follows. Among all the edges of $\mathcal{T}_{x^{\star}}$ crossing the circle $S_{r}(o)$, whose mean number is of order $r$, a very small number of them belong to semiinfinite geodesics. This first limit immediately follows from the directional result $\mathbb{E} \chi_{r}(\hat{u}, c) \rightarrow 0$, 
since by isotropy $\mathbb{E} \chi_{r}=r \mathbb{E} \chi_{r}(\hat{u}, 2 \pi)$, for any $\hat{u} \in S^{1}$. To state a null limit for the expectation of $\chi_{r}(\hat{u}, 2 \pi)$, we follow the method developed in [2,5]. This method essentially relies on two ingredients: a local approximation (in distribution) of the tree $\mathcal{T}_{x^{\star}}$ far away from its root by a suitable directed forest and the a.s. absence of bi-infinite geodesics with asymptotic directions $\hat{u}$ and $-\hat{u}$ in this directed forest. Actually, this absence of bi-infinite geodesics is a consequence of the coalescence of all the semi-infinite geodesics with direction $\hat{u}$, that is, Theorem 1 .

Observe that thanks to the translation invariance of the graph $G(X)$, Theorem 2 remains true whatever the Poisson point $x$ of $X$ at which the considered tree is rooted.

The rest of the paper is organized as follows. In Section 2.1, the modified Euclidean FPP model is defined. Existence and uniqueness of its geodesics are discussed. Section 2.2 is devoted to the outline of the proof of Theorem 1: the Burton-Keane argument is applied to the modified FPP model. But the heart of the proof, i.e., Proposition 5, is established in Section 3. Finally, Section 4 provides a proof of Theorem 2.

\section{The Burton-Keane argument for non-backtracking FPP}

In this section, $G(X)$ still denotes the Delaunay triangulation $\operatorname{Del}(X)$ and the relative neighborhood graph $\operatorname{Rng}(X)$ defined on the Poisson point process $X$. Our goal is to apply the BurtonKeane argument to a modified Euclidean FPP model defined as follows.

\subsection{The modified Euclidean FPP model}

Let $r \in \mathbb{R}$ and let us consider a modified FPP model on the graph $G(X)$ in which the length of an oriented path is given by its Euclidean length, unless it crosses the vertical line $l_{r}^{\vee}=\{r\} \times \mathbb{R}$ from right to left. In the latter case, its length is defined to be infinite. This is the reason why from now on, we have to consider oriented paths.

Let $\gamma=\left(x_{1}, \ldots, x_{k}\right)$ be an oriented path in $G(X)$ where $x_{1}, \ldots, x_{k}$ are Poisson points. Put

$$
v_{1}^{(r)}(\gamma)= \begin{cases}\infty, & \text { if } \pi_{1}\left(x_{j}\right) \geq r \geq \pi_{1}\left(x_{j+1}\right) \text { for some } j \in\{0, \ldots, k-1\}, \\ v_{1}(\gamma), & \text { otherwise, }\end{cases}
$$

where $\pi_{1}: \mathbb{R}^{2} \rightarrow \mathbb{R}$ denotes the projection onto the first coordinate. As before, an oriented path $\gamma=\left(x_{j}\right)_{1 \leq j \leq k}$ with $k \in \mathbb{N} \cup\{\infty\}$ on $G(X)$ is called geodesic with respect to $v_{1}^{(r)}$ if

$$
v_{1}^{(r)}(\gamma)=\inf \left\{v_{1}^{(r)}\left(\gamma^{\prime}\right): \gamma^{\prime} \text { is an oriented path on } G(X) \text { from } x_{1} \text { to } x_{k}\right\},
$$

where $\gamma\left[x_{i}, x_{j}\right]$ denotes the (oriented) sub-path of $\gamma$ from $x_{i}$ to $x_{j}$. We also make the convention that an oriented path crossing the vertical line $l_{r}^{\mathrm{v}}$ from right to left cannot be a geodesic.

Let us remark that geodesics of the modified FPP model, that is, w.r.t. $v_{1}^{(r)}$, can be markedly different from the ones of the original FPP model. To see it, let us consider $x, x^{\prime} \in X$ such that $\pi_{1}(x)<r<\pi_{1}\left(x^{\prime}\right)$ and let $\gamma$ be the geodesic connecting $x$ and $x^{\prime}$ in the original FPP model. Then, the geodesic w.r.t. $v_{1}^{(r)}$ from $x$ to $x^{\prime}$ is equal to $\gamma$ (up to the orientation) if and only if $\gamma$ 
crosses the vertical line $l_{r}^{\mathrm{v}}$ only one time. Besides, the geodesic w.r.t. $v_{1}^{(r)}$ from $x^{\prime}$ to $x$ do not exist. Also, let us remark in the relative neighborhood graph $\operatorname{Rng}(X)$ it can happen that the only edge starting from $x$ crosses the vertical line $l_{r}^{v}$ from right to left. In this case, there is no (finite or not) geodesic starting at $x$ in the modified FPP model. Such pathological situations do not occur in the Delaunay triangulation $\operatorname{Del}(X)$. Besides, in the modified FPP model on $\operatorname{Del}(X)$, it should be possible to prove existence of $\hat{u}$-unigeodesics for any asymptotic direction $\hat{u}$ such that $\left\langle\hat{u}, e_{1}\right\rangle \geq 0$ and starting from any vertex $x$, using [12], Proposition 2.8. However, this would still require some effort and will not be needed in the following.

Unlike existence, uniqueness of $\hat{u}$-unigeodesics - when they exist - in the modified FPP model is established fairly easily. Indeed, the classical argument due to Licea and Newman [15] shows that if $\hat{u} \in S^{1}$ is chosen suitably, then for each vertex $x \in G(X)$ there exists at most one semiinfinite geodesic w.r.t. $v_{1}^{(r)}$ with asymptotic direction $\hat{u}$ and starting point $x$. When it exists, it will be denoted $\gamma_{x}^{(r)}$.

To make the presentation self-contained, we reproduce from [15], Theorem 0 , the original argument for the uniqueness of $\hat{u}$-unigeodesics. See also [11], Lemma 6 , for another account. Let $D_{r}(\hat{u})$ be the event that for every $x \in X$ there exists at most one $\hat{u}$-unigeodesic w.r.t. $v_{1}^{(r)}$ starting from $x$. By stationarity, the probability of the event $D_{r}(\hat{u})$ does not depend on the value of $r \in \mathbb{R}$.

Lemma 3. It holds that $\int_{S^{1}} \mathbb{P}\left(D_{0}(\hat{u})^{c}\right) \mathrm{d} \hat{u}=0$ where $\mathrm{d} \hat{u}$ denotes the Lebesgue measure on $S^{1}$. In other words, for almost every $\hat{u} \in S^{1}, \mathbb{P}\left(D_{0}(\hat{u})\right)=1$.

Proof. If $D_{0}(\hat{u})$ does not occur, then there exists some point $x \in X$ featuring two $\hat{u}$-unigeodesics $\gamma_{1}$ and $\gamma_{2}$ that have $x$ as their only common point. Let $x_{1}, x_{2}$ be the respective successors of $x$ in these geodesics. Writing $\gamma_{+}(x), \gamma_{-}(x)$ for the trigonometrically highest and lowest geodesics w.r.t. $v_{1}^{(0)}$ in $G(X)$ starting from $x \in X$, we conclude from the planarity of $G(X)$ that at least one of $\gamma_{+}\left(x_{1}\right), \gamma_{+}\left(x_{2}\right), \gamma_{-}\left(x_{1}\right)$ and $\gamma_{-}\left(x_{2}\right)$ is trapped between $\gamma_{1}$ and $\gamma_{2}$. Hence, its asymptotic direction is also given by $\hat{u}$. In other words, we have $D_{0}(\hat{u})^{c} \subset H(\hat{u})$, where $H(\hat{u})$ denotes the event that there exists $x \in X$ such that $\gamma_{-}(x)$ or $\gamma_{+}(x)$ has asymptotic direction $\hat{u}$. Since in each realization, the event $H(\hat{u})$ can occur only for two directions of $S^{1}$, we deduce that $\int_{S^{1}} \mathbb{1}\{H(\hat{u})\} \mathrm{d} \hat{u}=0$. Since the asymptotic directions (if they exist) of $\gamma_{-}(x)$ and $\gamma_{+}(x)$ are measurable in the $\sigma$-algebra generated by $X$, the event $H(\hat{u})$ is jointly measurable in $\omega$ and $\hat{u}$. Hence, by Fubini's theorem,

$$
\int_{S^{1}} \mathbb{P}\left(D_{0}(\hat{u})^{c}\right) \mathrm{d} \hat{u} \leq \int_{S^{1}} \mathbb{P}(H(\hat{u})) \mathrm{d} \hat{u}=\mathbb{E} \int_{S^{1}} \mathbb{1}\{H(\hat{u})\} \mathrm{d} \hat{u}=0,
$$

as required.

In the following, we choose a direction $\hat{u}_{0} \in S^{1}$ such that $\mathbb{P}\left(D_{0}\left(\hat{u}_{0}\right)\right)=1$ and the absolute value of the angle of $\hat{u}_{0}$ with the $x$-axis is at most $\delta$, where $\delta>0$ is assumed to be the inverse of a sufficiently large integer, which is fixed in the entire manuscript. 


\subsection{Outline of the proof of Theorem 1}

Next, we introduce an event $F_{M}$ which is central in our study. It describes the existence of a distinguished geodesic in the modified FPP model that is protected from coalescing with other distinguished geodesics. To make this precise, we require additional notations. Given a semiinfinite path $\gamma$ of $G(X)$ and a point $P \in \gamma$, we denote by $\gamma[P]$ the semi-infinite subpath of $\gamma$ starting at $P$. Let $H^{-}=(-\infty, 0] \times \mathbb{R}$ and $H^{+}=[0, \infty) \times \mathbb{R}$ be the negative and positive vertical half-plane, respectively. We also denote by $C_{\delta}(P)$ the cone with apex $P \in \mathbb{R}^{2}$, asymptotic direction $\hat{u}_{0}$ and opening angle $\delta$. Let $A \oplus B=\{a+b: a \in A, b \in B\}$ denote the Minkowski sum of $A, B \subset \mathbb{R}^{2}$.

Definition 4. For $M>0$, let $F_{M}$ be the event meaning that there exists points $x_{\mathrm{m}}^{-} \in X \cap H^{-}$and $x_{\mathrm{m}}^{+} \in X \cap H^{+}$with the following properties:

(i) $\left[x_{\mathrm{m}}^{-}, x_{\mathrm{m}}^{+}\right]$forms an edge in $G(X)$ and is contained in $B_{M / 2}(o)$,

(ii) $\gamma_{\mathrm{m}}=\gamma_{x_{\mathrm{m}}^{+}}^{(0)}$ exists and is contained in the dilated cone $C_{\delta}(Q) \oplus B_{\delta M}(o)$, where $\{Q\}=$ $\left[x_{\mathrm{m}}^{-}, x_{\mathrm{m}}^{+}\right] \cap l_{0}^{\mathrm{v}}$,

(iii) if $z \in 4 \mathbb{Z} \times 2 \mathbb{Z}$ is such that $z \neq o$ and $\pi_{1}(z) \leq 0$, and $x^{-} \in X \cap\left(M z+H^{-}\right)$as well as $x^{+} \in X \cap\left(M z+H^{+}\right)$are such that

(a) $\left[x^{-}, x^{+}\right]$forms an edge in $G(X)$ and is contained in $B_{M / 2}(M z)$,

(b) $\gamma=\gamma_{x^{+}}^{\left(M \pi_{1}(z)\right)}$ exists,

then $\gamma[P] \cap \gamma_{\mathrm{m}}=\varnothing$, where $P$ denotes the last intersection point of $\gamma$ and $l_{0}^{\mathrm{v}}$. If such $P$ does not exist, we put $\gamma[P]=\gamma$.

Figure 2 provides an illustration of the event $F_{M}$. It is worth pointing out here that the geodesics involved in $F_{M}$ are w.r.t. $v_{1}^{(r)}$ for different values of $r$.

We now consider the family of $\hat{u}_{0}$-unigeodesics $\left\{\gamma_{x}\right\}_{x \in X}$ in the original FPP model on $G(X)$. This family can be used to define a forest $\mathcal{F}=\mathcal{F}_{\hat{u}_{0}}$ with vertex set $X$ by drawing an edge from $x$ to $y$ if $[x, y]$ is the first edge in the geodesic $\gamma_{x}$. If $N$ denotes the number of connected components in this forest, then Theorem 1 is equivalent to the assertion that $\mathbb{P}(N \geq 2)=0$. Of course, by isotropy, we could choose $\hat{u}_{0}=e_{1}$, but for our argument it will be notationally convenient to have a certain flexibility in the choice of the direction. We will always assume that the angle between $\hat{u}_{0}$ and $e_{1}$ is at most $\delta$.

The heart of our paper is to show that the event $F_{M}$ occurs with positive probability if the event $\{N \geq 2\}$ does so. In other words, from the hypothesis that the original FPP model contains noncoalescing $\hat{u}_{0}$-unigeodesics, we exhibit non-coalescing and non-backtracking (w.r.t. to different vertical lines) $\hat{u}_{0}$-unigeodesics so that the event $F_{M}$ occurs. Section 3 is devoted to its proof.

Proposition 5. If $\mathbb{P}(N \geq 2)>0$, then $\mathbb{P}\left(F_{M}\right)>0$ for $M$ large enough.

Although the event $F_{M}$ involves $\hat{u}_{0}$-unigeodesics w.r.t. $v_{1}^{(r)}$, for different values of $r$, these geodesics do not overlap and then leave any large rectangle via different edges. Hence, in contrast to Proposition 5, the classical argument [15], Theorem 1, applies without substantial further complications and leads to: 


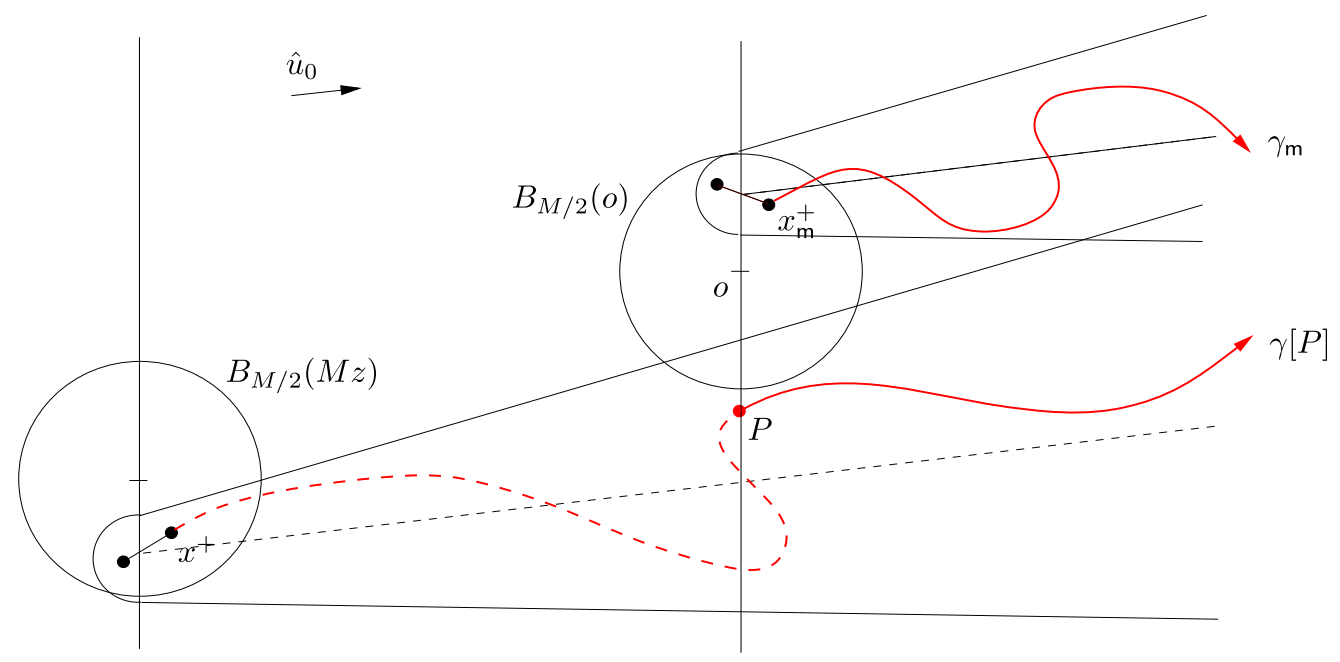

Figure 2. This picture represents the event $F_{M}$. The solid red curves are the geodesics $\gamma_{\mathrm{m}}=\gamma_{x_{\mathrm{m}}^{+}}^{(0)}$ and $\gamma[P]$ where $\gamma=\gamma_{x^{+}}^{\left(M \pi_{1}(z)\right)}$. They are both contained in the corresponding dilated cones and they do not overlap. Let us remark that for clarity, this picture does not respect the fact that $z$ belongs to $4 \mathbb{Z} \times 2 \mathbb{Z}$.

Proposition 6. It holds that $\mathbb{P}\left(F_{M}\right)=0$, for $M$ large enough.

In particular, Theorem 1 is a consequence of Propositions 5 and 6.

Proof of Proposition 6. In order to derive a contradiction, we assume that there would exist $M \geq 1$ with $\mathbb{P}\left(F_{M}\right)>0$. For $L \geq 1$ let $f(L)$ denote the number of $z \in(4 \mathbb{Z} \cap[-4 L, 4 L]) \times$ $(2 \mathbb{Z} \cap[-2 L, 2 L])$ such that $(X-M z) \in F_{M}$. Then, by stationarity of $G(X)$ and the choice of $M$, the first moment of $f(L)$ grows quadratically in $L$. On the other hand, let $f^{\prime}(L)$ denote the number of edges in $G(X)$ intersecting the boundary of $[-8 L M, 8 L M] \times[-4 L M, 4 L M]$. Then $\mathbb{E} f^{\prime}(L)$ grows linearly in $L$. Hence, it suffices to show that if $L$ is sufficiently large, then $f(L) \leq f^{\prime}(L)$ holds almost surely.

In order to prove this claim, let $z, z^{\prime} \in(4 \mathbb{Z} \cap[-4 L, 4 L]) \times(2 \mathbb{Z} \cap[-2 L, 2 L])$ be such that $z \neq z^{\prime},(X-M z) \in F_{M}$ and $\left(X-M z^{\prime}\right) \in F_{M}$. In particular, the definition of $F_{M}$ provides us with points $x_{z, \mathrm{~m}}^{-} \in X \cap\left(M z+H^{-}\right), x_{z, \mathrm{~m}}^{+} \in X \cap\left(M z+H^{+}\right)$and $x_{z^{\prime}, \mathrm{m}}^{-} \in X \cap\left(M z^{\prime}+H^{-}\right)$, $x_{z^{\prime}, \mathrm{m}}^{+} \in X \cap\left(M z^{\prime}+H^{+}\right)$satisfying the properties asserted in that definition. To simplify notation, we put

$$
\gamma_{z, \mathrm{~m}}=\gamma_{x_{z, \mathrm{~m}}^{+}}^{\left(M \pi_{1}(z)\right)} \text { and } \quad \gamma_{z^{\prime}, \mathrm{m}}=\gamma_{z_{z^{\prime}, \mathrm{m}}^{+}}^{\left(M \pi_{1}\left(z^{\prime}\right)\right)}
$$

Then, we claim that $\gamma_{z, \mathrm{~m}}$ and $\gamma_{z^{\prime}, \mathrm{m}}$ do not leave the rectangle $[-8 L M, 8 L M] \times[-4 L M, 4 L M]$ via the same edge. Indeed, without loss of generality, we may assume that $\pi_{1}(z) \leq \pi_{1}\left(z^{\prime}\right)$. If $\pi_{1}(z)=\pi_{1}\left(z^{\prime}\right)$, then we know from the definition of $F_{M}$ that $\gamma_{z, \mathrm{~m}}$ and $\gamma_{z^{\prime}, \mathrm{m}}$ are disjoint. Hence, we may assume that $\pi_{1}(z)<\pi_{1}\left(z^{\prime}\right)$. Since $X-M z \in F_{M}$, the geodesic $\gamma_{z, \mathrm{~m}}$ is contained in the 


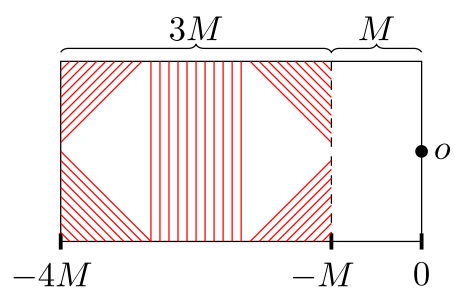

Figure 3. Edges in the Delaunay triangulation in the event $\left\{X \cap R_{M}^{-}=\varnothing\right\} \cap\left\{X^{(1)} \in A_{M}^{\prime}\right\}$.

cone $C_{\delta}(Q) \oplus B_{\delta M}(M z)$, where $\{Q\}=\left[x_{z, \mathrm{~m}}^{-}, x_{z, \mathrm{~m}}^{+}\right] \cap l_{M z}^{\mathrm{v}}$. Hence, for small $\delta$ we conclude that the last intersection point $P$ of $\gamma_{z, \mathrm{~m}}$ with the vertical line $l_{M z^{\prime}}^{\mathrm{v}}$ is contained in $[-8 L M, 8 L M] \times$ $[-4 L M, 4 L M]$. Now, the occurrence of the event $F_{M}$ implies that $\gamma_{z, \mathrm{~m}}[P]$ and $\gamma_{z^{\prime}, \mathrm{m}}$ are disjoint, so that they leave the rectangle $[-8 L M, 8 L M] \times[-4 L M, 4 L M]$ via different edges. Let us add that the intersection point $P$ could be outside $[-4 L M, 4 L M] \times[-2 L M, 2 L M]$, but necessarily inside the large rectangle $[-8 L M, 8 L M] \times[-4 L M, 4 L M]$ for $\delta$ small enough. This is the reason why $f^{\prime}(L)$ counts the edges intersecting the boundary of $[-8 L M, 8 L M] \times[-4 L M, 4 L M]$.

\section{Proof of Proposition 5}

Since the proof of Proposition 5 is rather long, we provide the reader with a brief outlook. We are going to define three events $E_{M}, A_{M}^{\prime}$ and $A_{M}^{\prime \prime}$ such that their intersection implies $F_{M}$ and occurs with positive probability, therefore proving Proposition 5.

The event $E_{M}$, defined in Section 3.1, mainly ensures the existence of three disjoint geodesics $\gamma_{\mathrm{u}}, \gamma_{\mathrm{m}}$ and $\gamma_{\mathrm{d}}$, all starting from the segment $\{0\} \times[-\delta M, \delta M]$ and included in $H^{+}$. As in the classic Burton-Keane argument, the role of $\gamma_{\mathrm{u}}$ and $\gamma_{\mathrm{d}}$ is to protect $\gamma_{\mathrm{m}}$ from above and below, respectively. In Lemma 7 , it is proved that the event $\left\{X \in E_{M}\right\}$ occurs with high probability whenever $\mathbb{P}(N \geq 2)>0$.

To protect $\gamma_{\mathrm{m}}$ from the left, we turn the $(4 M \times 2 M)$-box $R_{M}=[-4 M, 0] \times[-M, M]$ into an obstacle. To do it, we first assume that the Poisson point process $X$ does not contain any points in the $(3 M \times 2 M)$-box $R_{M}^{-}=[-4 M,-M] \times[-M, M]$; we set $X^{(1)}=X \backslash R_{M}^{-}$. This results in a structural change of the graph inside $R_{M}^{-}$, which is illustrated in Figure 3 . To ensure that the sketch in Figure 3 is accurate (see Lemma 10 of Section 3.2), we need to make some assumptions on the configuration of $X$ outside $R_{M}^{-}$. This is the role of the event $A_{M}^{\prime}$. First, Lemma 12 says that $\mathbb{P}\left(X^{(1)} \in A_{M}^{\prime}\right)$ tends to 1 as $M \rightarrow \infty$. Moreover, under the event $A_{M}^{\prime}$, the replacement of $X$ with $X^{(1)}$ causes modifications in the graph only inside the half-plane $H^{-}$(see Lemma 9). Now, the occurrence of the event $E_{m}$ is not influenced by such modifications because $E_{m}$ only concerns geodesics w.r.t. $v_{1}^{(0)}$. As a consequence,

$$
\mathbb{P}\left(X^{(1)} \in E_{M} \cap A_{M}^{\prime}\right)=\mathbb{P}\left(X \in E_{M}, X^{(1)} \in A_{M}^{\prime}\right),
$$

which then tends to 1 as $M$ tends to infinity. 
Third, we need a certain control over lengths of geodesics in $G(X)$. This property will be encoded in an event $A_{M}^{\prime \prime}$ defined in Section 3.3 that only depends on the configuration of $X$ in $\mathbb{R}^{2} \backslash R_{M}^{-}$. We will see in Lemma 17 that $\mathbb{P}\left(X^{(1)} \in A_{M}^{\prime \prime}\right)$ tends to 1 as $M \rightarrow \infty$.

Finally, the proof of Proposition 5 ends with Section 3.4 in which a key deterministic argument ensures that $X^{(1)} \in E_{M} \cap A_{M}^{\prime} \cap A_{M}^{\prime \prime}$ almost surely implies that $X^{(1)} \in F_{M}$. See Lemma 18 . Hence, we conclude by the following computation:

$$
\begin{aligned}
0 & <\mathbb{P}\left(X^{(1)} \in E_{M} \cap A_{M}^{\prime} \cap A_{M}^{\prime \prime}\right) \mathbb{P}\left(X \cap R_{M}^{-}=\varnothing\right) \\
& =\mathbb{P}\left(X^{(1)} \in E_{M} \cap A_{M}^{\prime} \cap A_{M}^{\prime \prime}, X \cap R_{M}^{-}=\varnothing\right) \\
& \leq \mathbb{P}\left(X^{(1)} \in F_{M}, X \cap R_{M}^{-}=\varnothing\right) \\
& \leq \mathbb{P}\left(X \in F_{M}\right) .
\end{aligned}
$$

\subsection{The event $E_{M}$}

In this section, using the assumption that $\mathbb{P}(N \geq 2)>0$, we explain how to protect the geodesic $\gamma_{\mathrm{m}}$ from above and below by two semi-infinite geodesics $\gamma_{\mathrm{u}}$ and $\gamma_{\mathrm{d}}$ which do not coalesce with $\gamma_{\mathrm{m}}$. Note that by ergodicity, our assumption implies that $\mathbb{P}(N \geq 2)=1$.

As the main result of this section, we can construct the desired distinguished geodesics, which are considered as subsets of the random closed set of segments $\mathcal{E}(X)$ describing the edges of $G(X)$. The geodesics $\gamma_{\mathrm{u}}, \gamma_{\mathrm{m}}$ and $\gamma_{\mathrm{d}}$ intersect the interval $\{0\} \times[-\delta M, \delta M]$ in points $P_{\mathrm{u}}, P_{\mathrm{m}}$ and $P_{\mathrm{d}}$. The subsequent vertices in the geodesic are called $x_{\mathrm{u}}^{+}, x_{\mathrm{m}}^{+}$and $x_{\mathrm{d}}^{+}$. More precisely, for $M \geq 1$, we let $E_{M}$ denote the event that there exist

$$
P_{\mathrm{u}}, P_{\mathrm{m}}, P_{\mathrm{d}} \in \mathcal{E}(X) \cap(\{0\} \times[-\delta M, \delta M]),
$$

such that

(i) $\pi_{2}\left(P_{\mathrm{u}}\right)>\pi_{2}\left(P_{\mathrm{m}}\right)>\pi_{2}\left(P_{\mathrm{d}}\right)$, and

(ii) the geodesics $\left\{\gamma_{\sigma}\right\}_{\sigma \in\{\mathrm{u}, \mathrm{m}, \mathrm{d}\}}=\left\{\gamma_{x_{\sigma}^{+}}^{(0)}\right\}_{\sigma \in\{\mathrm{u}, \mathrm{m}, \mathrm{d}\}}$ exist and are disjoint, and

(iii) for each $\sigma \in\{\mathrm{u}, \mathrm{m}, \mathrm{d}\}$ the geodesic $\gamma_{\sigma}$ is contained in the dilated cone $C_{\delta}\left(P_{\sigma}\right) \oplus B_{\delta M}(o)$.

Now, we show that the event $E_{M}$ occurs with high probability.

Lemma 7. If $\mathbb{P}(N \geq 2)>0$, then $\lim _{M \rightarrow \infty} \mathbb{P}\left(E_{M}\right)=1$.

Proof. As a first step, we consider geodesics that do not backtrack behind the vertical line $l_{0}^{v}$. More precisely, we define the linear point process $Y$ to consist of those $P \in l_{0}^{\vee}$ that can be represented as $P=\left[x^{-}, x^{+}\right] \cap l_{0}^{\mathrm{v}}$, where $x^{-}, x^{+} \in X$ are assumed to be connected by an edge in $G(X)$. Moreover, we put $\gamma_{P}=\gamma_{x^{+}}$, where we assume that $x^{+}$is chosen such that $\pi_{1}\left(x^{+}\right)>0$. Then, we let $Y^{\prime} \subset Y$ denote the thinning of $Y$ consisting of all $P \in Y$ for which the geodesic $\gamma_{P}$ is contained in the positive half-plane $H^{+}$. In the following, it will play an important role that if $\gamma_{P}$ is a geodesic in $G(X)$ with respect to $\nu_{1}$ and $P \in Y^{\prime}$, then $\gamma_{P}$ is also a geodesic with respect to $v_{1}^{(0)}$, i.e., $\gamma_{P}=\gamma_{P}^{(0)}$. Identifying $l_{0}^{\vee}$ with the real line, we think of $Y^{\prime}$ as one-dimensional 


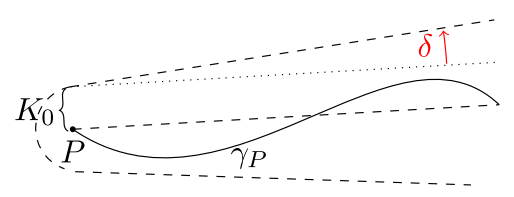

Figure 4. Illustration of the retention condition for $Y^{\prime \prime}$.

stationary and ergodic point process. Since $\gamma_{P}$ has asymptotic direction $\hat{u}_{0}$, the intensity of $Y^{\prime}$ is positive when regarded as one-dimensional point process. Indeed, if $P$ is any point on $Y$, then the last intersection point of $\gamma_{P}$ with $l_{0}^{\vee}$ belongs to $Y^{\prime}$.

In the following, it will be important to exert some control over the amount of fluctuation of the distinguished geodesics. More precisely, the definition of asymptotic direction shows that for every $P=\left[x^{-}, x^{+}\right] \cap l_{0}^{\vee} \in Y^{\prime}$ there exists a random $k>0$ such that $\left|x^{-}-x^{+}\right| \leq k$ and $\gamma_{P} \subset C_{\delta}(P) \oplus B_{k}(o)$. In particular, there exists a deterministic $K_{0}>0$ such that the thinning $Y^{\prime \prime}$ of $Y^{\prime}$ consisting of all $P=\left[x^{-}, x^{+}\right] \cap l_{0}^{\vee} \in Y^{\prime}$ such that $\left|x^{-}-x^{+}\right| \leq K_{0}$ and $\gamma_{P}$ is contained in the dilated cone $C_{\delta}(P) \oplus B_{K_{0}}(o)$ forms a stationary and ergodic point process with positive intensity. The retention condition for $Y^{\prime \prime}$ is illustrated in Figure 4.

First, we claim that for every $t \in \mathbb{R}$ the probability $q_{t}$ that the geodesics $\gamma_{P}$ coalesce for all $P \in Y^{\prime \prime} \cap(\{0\} \times[t, \infty))$ is equal to 0 . Indeed, stationarity implies that $q_{t}$ does not depend on $t$, so that by planarity $\lim _{t \rightarrow-\infty} q_{t} \leq \mathbb{P}(N \leq 1)=0$. Hence,

$$
\lim _{M \rightarrow \infty} \mathbb{P}\left(E_{M}^{\prime}\right)=1,
$$

where $E_{M}^{\prime}$ denotes the event that there exist $P, P^{\prime}, P^{\prime \prime} \in Y^{\prime \prime} \cap(\{0\} \times \mathbb{R})$ such that

(i) $\pi_{2}(P) \in\left[\frac{3}{4} \delta M, \delta M\right], \pi_{2}\left(P^{\prime}\right) \in\left[-\frac{1}{4} \delta M, \frac{1}{4} \delta M\right]$ and $\pi_{2}\left(P^{\prime \prime}\right) \in\left[-\delta M,-\frac{3}{4} \delta M\right]$, and

(ii) the geodesics $\gamma_{P}, \gamma_{P^{\prime}}$ and $\gamma_{P^{\prime \prime}}$ are pairwise non-coalescent.

Since $E_{M}^{\prime} \subset E_{M}$, this completes the proof.

The following result shows that Lemma 7 allows us to restrict our attention to potential coalescence of $\gamma_{\mathrm{m}}$ with geodesics that cross the segment $\{0\} \times[-\delta M, \delta M]$. Recall that if $\gamma$ is an arbitrary oriented path in $G(X)$ and $P \in \gamma$, then $\gamma[P]$ denotes the oriented subpath of $\gamma$ starting at $P$.

Lemma 8. Let $z \in 4 \mathbb{Z} \times 2 \mathbb{Z}$ be such that $z \neq o$ and $\pi_{1}(z) \leq 0$. Then, almost surely under the event $E_{M}$, the following assertion holds. If $x^{-} \in X \cap\left(M z+H^{-}\right)$and $x^{+} \in X \cap\left(M z+H^{+}\right)$are such that

(i) $\left[x^{-}, x^{+}\right]$forms an edge in $G(X)$ that is contained in $B_{M / 2}(M z)$,

(ii) $\gamma=\gamma_{x^{+}}^{\left(M \pi_{1}(z)\right)}$ exists, and

(iii) $\gamma[P] \cap \gamma_{\mathrm{m}} \neq \varnothing$, where $P$ denotes the last intersection point of $\gamma$ and $l_{0}^{\mathrm{v}}$,

then $\left|\pi_{2}(P)\right| \leq \delta M$. 
Proof. In order to derive a contradiction, we assume that $\left|\pi_{2}(P)\right|>\delta M$. Since $\gamma_{\mathrm{m}}$ is enclosed by the union of $\{0\} \times[-\delta M, \delta M],\left[x_{\mathrm{u}}^{-}, x_{\mathrm{u}}^{+}\right] \cup \gamma_{\mathrm{u}}$ and $\left[x_{\mathrm{d}}^{-}, x_{\mathrm{d}}^{+}\right] \cup \gamma_{\mathrm{d}}$, we deduce that $\gamma[P]$ has a common vertex with $\gamma_{\mathrm{u}}$ or $\gamma_{\mathrm{d}}$. We let $x$ be the last such point and assume that it lies on $\gamma_{\mathrm{u}}$. Then, $\gamma[x]$ and $\gamma_{u}[x]$ are two distinct $\hat{u}_{0}$-geodesics in $G(X)$ with respect to $v_{1}^{(0)}$, contradicting Lemma 3 and the choice of $\hat{u}_{0}$.

Remark 1. Note that if $\pi_{1}(z)=0$, then it is impossible to obtain $\left|\pi_{2}(P)\right| \leq \delta M$. Hence, in this case the event $E_{M}$ already suffices to ensure that $\gamma[P] \cap \gamma_{\mathrm{m}}=\varnothing$.

\subsection{The event $A_{M}^{\prime}$}

The second part of the shield is obtained by changing the Poisson point process in $(3 M \times 2 M)$ box $R_{M}^{-}=[-4 M,-M] \times[-M, M]$ so as to increase the cost of passing through the rectangle $R_{M}$. An important feature in our choice of the event $E_{M}$ is that it only involves geodesics in $G(X)$ with respect to $v_{1}^{(0)}$, but not $v_{1}$. Hence, if the modifications in $R_{M}^{-}$are organized such that they do not influence the configuration of $G(X)$ in $H^{+}$, then the occurrence of the event $E_{M}$ is not influenced by this modification. We stress that the latter implication was false if we considered geodesics in $G(X)$ with respect to $v_{1}$ and not $v_{1}^{(0)}$.

First, we introduce a family of events $\left\{A_{M, 1}^{\prime}\right\}_{M \geq 1}$ guaranteeing that changes of the Poisson point process within $R_{M}^{-}$do not influence the Delaunay triangulation or the relative neighborhood graph outside the dilated rectangle $R_{M}^{-} \oplus Q_{8 \varepsilon M}(o)$. Here we put $\varepsilon=\varepsilon(M)=M^{-31 / 32}$ and $Q_{8 \varepsilon M}(o)=[-4 \varepsilon M, 4 \varepsilon M]^{2}$. To be more precise, we subdivide $\left(R_{M}^{-} \oplus Q_{M}(o)\right) \backslash R_{M}^{-}$into

$$
K_{M}=\left(4 \varepsilon^{-1}\right)\left(3 \varepsilon^{-1}\right)-\left(3 \varepsilon^{-1}\right)\left(2 \varepsilon^{-1}\right)=6 \varepsilon^{-2}
$$

congruent subsquares $Q_{i}=Q_{\varepsilon M}\left(v_{i}\right)$ of side length $\varepsilon M$, where we assume that $\varepsilon^{-1}$ is an integer. Then, we write $\left\{X^{(1)} \in A_{M, 1}^{\prime}\right\}$ if $\#\left(X^{(1)} \cap Q_{i}\right) \in\left[1,2 \varepsilon^{2} M^{2}\right]$ holds for all $1 \leq i \leq K_{M}$. Since $\#\left(X^{(1)} \cap Q_{i}\right)$ is Poisson-distributed with parameter $\varepsilon^{2} M^{2}$, the probability of the event $A_{M, 1}^{\prime}$ tends to 1 as $M \rightarrow \infty$. Next, we show that under $\left\{X^{(1)} \in A_{M, 1}^{\prime}\right\}$ the Delaunay triangulation and the relative neighborhood graph exhibit stabilization outside $R_{M}^{-} \oplus Q_{8 \varepsilon M}(o)$.

Lemma 9. If $X^{(1)} \in A_{M, 1}^{\prime}$, then

$$
G\left(X^{(1)} \cup \psi\right) \backslash\left(R_{M}^{-} \oplus Q_{8 \varepsilon M}(o)\right)=G\left(X^{(1)}\right) \backslash\left(R_{M}^{-} \oplus Q_{8 \varepsilon M}(o)\right)
$$

holds for every finite point configuration $\psi$ in $R_{M}^{-}$.

Proof. We only provide the proof for the Delaunay triangulation, since the case of the relative neighborhood graph is similar but easier. In order to derive a contradiction, assume that we could find finite subsets $\psi, \psi^{\prime} \subset R_{M}^{-}$and an edge $e$ in $\operatorname{Del}\left(X^{(1)} \cup \psi\right)$ such that (i) at least one end point of $e$ is outside $R_{M}^{-} \oplus Q_{8 \varepsilon M}(o)$ and (ii) $e$ is not an edge in $\operatorname{Del}\left(X^{(1)} \cup \psi^{\prime}\right)$. Then, there exists a disk $D$ containing $e$ and no points of $X^{(1)} \cup \psi$ in its interior. Note that $D$ does not intersect $R_{M}^{-}$, since 
otherwise it would cover one of the cubes $Q_{i}$, contradicting the assumption that $X^{(1)} \cap Q_{i} \neq \varnothing$. Hence, $D \cap R_{M}^{-}=\varnothing$ and therefore $e$ is also an edge in $\operatorname{Del}\left(X^{(1)} \cup \psi^{\prime}\right)$.

Moreover, we show that under the event $\left\{A_{M, 1}^{\prime}\right\}_{M \geq 1}$ the sketch in Figure 3 is accurate. The vertical and diagonal edges correspond to parts (i) and (ii) of the following result.

Lemma 10. If $X^{(1)} \in A_{M, 1}^{\prime}$, then

(i) there exists an edge $[x, y]$ in $\operatorname{Del}\left(X^{(1)}\right)$ with $\max \left\{\left|\pi_{1}(x)+2 M\right|,\left|\pi_{1}(y)+2 M\right|\right\} \leq 8 \sqrt{\varepsilon} M$, $\pi_{2}(x) \geq M$ and $\pi_{2}(y) \leq-M$,

(ii) for every $\rho \in[8 \varepsilon, 1-8 \varepsilon]$ there exists an edge $[x, y]$ in $\operatorname{Del}\left(X^{(1)}\right)$ such that

(a) $\max \left\{\left|\pi_{1}(x)-(-1-\rho) M\right|,\left|\pi_{2}(y)-(1-\rho) M\right|\right\} \leq 8 \sqrt{\varepsilon} M$, and

(b) $0 \leq \pi_{2}(x)-M \leq 8 \varepsilon M$ and $0 \leq \pi_{1}(y)+M \leq 8 \varepsilon M$.

Proof. Fix $P_{0}=((-2-8 \varepsilon) M, 0)$ and consider the disk $D=B_{(1+8 \varepsilon) M}\left(P_{0}\right)$ of radius $M+8 \varepsilon M$. First, by the choice of the subsquares $Q_{i}$, there exist $x^{\prime}, y^{\prime} \in X^{(1)} \cap D$ with $\pi_{2}\left(x^{\prime}\right) \geq M$ and $\pi_{2}\left(y^{\prime}\right) \leq-M$. Conversely, we claim that if $x \in X^{(1)} \cap D$ and $\left|\pi_{2}(x)\right| \geq M$, then $\left|\pi_{1}(x)+2 M\right| \leq$ $8 \sqrt{\varepsilon} M$. Indeed,

$$
\begin{aligned}
8 \varepsilon M & \geq\left|x-P_{0}\right|-M=\frac{\left(\pi_{1}(x)-(-2-8 \varepsilon) M\right)^{2}+\pi_{2}(x)^{2}-M^{2}}{\sqrt{\left(\pi_{1}(x)-(-2-8 \varepsilon) M\right)^{2}+\pi_{2}(x)^{2}}+M} \\
& \geq \frac{\left(\pi_{1}(x)-(-2-8 \varepsilon) M\right)^{2}}{3 M},
\end{aligned}
$$

so that

$$
\left|\pi_{1}(x)+2 M\right| \leq\left|\pi_{1}(x)-(-2-8 \varepsilon) M\right|+8 \varepsilon M \leq 2 \sqrt{6 \varepsilon} M+8 \varepsilon M,
$$

which is smaller than $8 \sqrt{\varepsilon} M$ if $M$ is sufficiently large. Now, we let $D^{\prime}$ denote the smallest disk with center $P_{0}$ that contains both at least one $x \in X^{(1)}$ with $\pi_{2}(x) \geq M$ and at least one $y \in X^{(1)}$ with $\pi_{2}(y) \leq-M$. Without loss of generality, we may assume $y$ to be the only point with in $D^{\prime}$ with that property. Finally, we consider the smallest disk $D^{\prime \prime}$ with center on the segment $\left[P_{0}, y\right]$ that has $y$ on its boundary and still contains at least one $x \in X^{(1)}$ with $\pi_{2}(x) \geq M$. Then, there is an edge between $x$ and $y$ in $\operatorname{Del}\left(X^{(1)}\right)$.

For the second claim, we proceed similarly, but for the convenience of the reader, we provide some details. For $\rho \in[8 \varepsilon, 1-8 \varepsilon]$, we fix $P_{0}=((-1-\rho) M,(1-\rho) M)$ and consider the disk $D=B_{\rho M+8 \varepsilon M}\left(P_{0}\right)$ of radius $\rho M+8 \varepsilon M$ centered at $P_{0}$. Again, the choice of the subsquares $Q_{i}$ implies that there exist $x^{\prime}, y^{\prime} \in X^{(1)} \cap D$ with $\pi_{2}\left(x^{\prime}\right) \geq M$ and $\pi_{1}\left(y^{\prime}\right) \geq-M$. Conversely, we claim that if $x \in X^{(1)} \cap D$ and $\pi_{2}(x) \geq M$, then $\left|\pi_{1}(x)+(1+\rho) M\right| \leq 4 \sqrt{\varepsilon} M$. Indeed, as before,

$$
\begin{aligned}
8 \varepsilon M & \geq \frac{\left(\pi_{1}(x)-(-1-\rho) M\right)^{2}+\left(\pi_{2}(x)-(1-\rho) M\right)^{2}-\rho^{2} M^{2}}{\sqrt{\left(\pi_{1}(x)-(-1-\rho) M\right)^{2}+\left(\pi_{2}(x)-(1-\rho) M\right)^{2}}+\rho M} \\
& \geq \frac{\left(\pi_{1}(x)-(-1-\rho) M\right)^{2}}{2 M},
\end{aligned}
$$


so that

$$
\left|\pi_{1}(x)-(-1-\rho) M\right| \leq 4 \sqrt{\varepsilon} M .
$$

By an analogous computation, we see that if $y \in X^{(1)} \cap D$ and $\pi_{1}(y) \geq-M$, then $\mid \pi_{2}(y)-$ $(1-\rho) M \mid \leq 4 \sqrt{\varepsilon} M$. Similar as in the previous case, shrinking and shifting $D$ until it contains precisely one $x \in X^{(1)}$ with $\pi_{2}(x) \geq M$ and one $y \in X^{(1)}$ with $\pi_{1}(y) \geq-M$ constructs the desired edge.

Remark 2. In particular, part (i) shows that under the event $A_{M, 1}^{\prime}$, if $\gamma$ is a path in $G(X)$ that starts in $H^{-} \backslash R_{M}$, intersects the segment $\{0\} \times[-\delta M, \delta M]$ but does not intersect the area $[(-1+$ $8 \sqrt{\varepsilon}) M, 0] \times(\mathbb{R} \backslash[-M, M])$, then $\gamma$ intersects the union of segments $[(-2-8 \sqrt{\varepsilon}) M,(-1+$ $8 \sqrt{\varepsilon}) M] \times\{ \pm M\}$.

Moreover, we obtain very precise control over the behavior of edges in $\operatorname{Del}\left(X^{(1)}\right)$ intersecting the horizontal segment $[-2 M,-M] \times\{M\}$.

Corollary 11. Assume $X^{(1)} \in A_{M, 1}^{\prime}$ and that $e$ is an edge of $\operatorname{Del}\left(X^{(1)}\right)$ intersecting the segment $[(-2+\delta) M,(-1-\delta) M] \times\{M\}$ at some point $P_{1}$ and define $\rho$ such that $\pi_{1}\left(P_{1}\right)=(-1-\rho) M$. Then, $e$ intersects the vertical segment

$$
\{-M\} \times[(1-\rho-32 \sqrt{\varepsilon}) M,(1-\rho+32 \sqrt{\varepsilon}) M],
$$

and therefore the length of $e$ is bounded below by $(\sqrt{2} \rho-32 \sqrt{\varepsilon}) M$.

Proof. By Lemma 10 there exist $x, x^{\prime}, y, y^{\prime} \in X$ such that $[x, y]$ and $\left[x^{\prime}, y^{\prime}\right]$ are edges in $\operatorname{Del}(X)$ with the following properties.

(i) $\max \left\{\left|\pi_{1}(x)-(-1-\rho-16 \sqrt{\varepsilon}) M\right|,\left|\pi_{2}(y)-(1-\rho-16 \sqrt{\varepsilon}) M\right|\right\} \leq 8 \sqrt{\varepsilon} M$,

(ii) $\max \left\{\left|\pi_{1}\left(x^{\prime}\right)-(-1-\rho+16 \sqrt{\varepsilon}) M\right|,\left|\pi_{2}\left(y^{\prime}\right)-(1-\rho+16 \sqrt{\varepsilon}) M\right|\right\} \leq 8 \sqrt{\varepsilon} M$,

(iii) $\pi_{2}(x), \pi_{2}\left(x^{\prime}\right) \in[M,(1+8 \varepsilon) M]$ and $\pi_{1}(y), \pi_{1}\left(y^{\prime}\right) \in[-M,(-1+8 \varepsilon) M]$.

If we let $P_{2}$ denote the intersection point of $[x, y]$ with the horizontal segment $[-2 M,-M] \times$ $\{M\}$, then

$$
\left(\pi_{1}\left(P_{2}\right)-(-1-\rho) M\right) \in[-26 \sqrt{\varepsilon} M,-6 \sqrt{\varepsilon} M] .
$$

Similarly, if $P_{2}^{\prime}$ denotes the intersection point of $\left[x^{\prime}, y^{\prime}\right]$ with the horizontal segment $[-2 M,-M]$, then

$$
\left(\pi_{1}\left(P_{2}^{\prime}\right)-(-1-\rho) M\right) \in[6 \sqrt{\varepsilon} M, 26 \sqrt{\varepsilon} M] .
$$

As similar relations can be obtained for the intersections $P_{3}, P_{3}^{\prime}$ of $[x, y]$ and $\left[x^{\prime}, y^{\prime}\right]$ with the vertical segment $\{-M\} \times[0, M]$, we see that $e$ is trapped between $[x, y]$ and $\left[x^{\prime}, y^{\prime}\right]$. In particular, $e$ intersects the vertical segment $\{-M\} \times\left(\pi_{2}\left(P_{3}\right), \pi_{2}\left(P_{3}^{\prime}\right)\right)$. Since

$$
(1-\rho-26 \sqrt{\varepsilon}) M \leq \pi_{2}\left(P_{3}\right) \leq \pi_{2}\left(P_{3}^{\prime}\right) \leq(1-\rho+26 \sqrt{\varepsilon}) M,
$$

this completes the proof. 
Remark 3. A similar result holds if $e$ intersects the segment $[(-2-\delta) M,(-2+\delta) M] \times\{M\}$. However, for those edges there are two options. Either they intersect the vertical segment $\{-M\} \times$ $\mathbb{R}$ close to the point $(-M, 0)$ or they intersect the horizontal segment $\mathbb{R} \times\{-M\}$ close to the point $(-2 M,-M)$.

Remark 4. Note that if $X^{(1)} \in A_{M, 1}^{\prime}$, then $\operatorname{Rng}\left(X^{(1)}\right)$ does not contain an edge intersecting the segment $[(-2-\delta) M,(-1-\delta) M] \times\{M\}$. Indeed, if $[x, y]$ was an edge of this type, then it follows from Corollary 11 that at least one of the subsquares $Q_{\varepsilon M}\left(v_{i}\right)$ introduced in the paragraph preceding Lemma 9 satisfies $Q_{\varepsilon M}\left(v_{i}\right) \subset B_{|x-y|}(x) \cap B_{|x-y|}(y)$. In particular, under the event $A_{M, 1}^{\prime}$ the segment $[x, y]$ does not form an edge in $\operatorname{Rng}\left(X^{(1)}\right)$.

In addition to the stabilization property, it will also be important to know that shortest pathlengths of $G\left(X^{(1)}\right)$ in the annulus $\left(R_{M}^{-} \oplus Q_{M}(o)\right) \backslash R_{M}^{-}$are not too long. Since shortestpath lengths in the relative neighborhood graph are closely related to descending chains [1], Lemma 10, we first recall the notion of descending chains from [7]. For $b \geq 1$ we say that a sequence of distinct vertices $X_{i_{1}}, \ldots, X_{i_{n}} \in X^{(1)}$ forms a $b$-bounded descending chain if

$$
b \geq\left|X_{i_{1}}-X_{i_{2}}\right| \geq \cdots \geq\left|X_{i_{n-1}}-X_{i_{n}}\right| .
$$

Now, we say that the event $A_{M, 2}^{\prime}$ occurs if every $16 \varepsilon M$-bounded descending chain of $X^{(1)}$ starting in $R_{M}^{-} \oplus Q_{M}(o)$ consists of at most $10^{4} \varepsilon^{2} M^{2}$ hops. Finally, we put $A_{M}^{\prime}=A_{M, 1}^{\prime} \cap A_{M, 2}^{\prime}$. Using the results from [7], we show that the events $A_{M}^{\prime}$ occur with high probability.

Lemma 12. It holds that $\lim _{M \rightarrow \infty} \mathbb{P}\left(X^{(1)} \in A_{M}^{\prime}\right)=1$.

Proof. Since we have seen that $\mathbb{P}\left(A_{M, 1}^{\prime}\right)$ tends to 1 as $M \rightarrow \infty$, it remains to show that $\lim _{M \rightarrow \infty} \mathbb{P}\left(A_{M, 2}^{\prime}\right)=1$. First, monotonicity of descending chains allows to reduce the claim to the computation of the probability that in a homogeneous Poisson point process, every $16 \varepsilon M$ bounded descending chain starting in $R_{M}^{-} \oplus Q_{M}(o)$ consists of at most $10^{4} \varepsilon^{2} M^{2}$ hops. But then the computations in [7], Section 3.2, show that the probability of obtaining a $16 \varepsilon M$-bounded descending chain of $X^{(1)}$ starting in $R_{M}^{-} \oplus Q_{M}(o)$ and consisting of more than $10^{4} \varepsilon^{2} M^{2}$ hops is at most

$$
\nu_{2}\left(R_{M}^{-} \oplus Q_{M}(o)\right) \frac{\left(16^{2} \pi \varepsilon^{2} M^{2}\right)^{10^{4} \varepsilon^{2} M^{2}}}{\left(10^{4} \varepsilon^{2} M^{2}\right) !},
$$

where $v_{2}$ denotes the Lebesgue measure. By Stirling's formula, this expression tends to 0 as $M \rightarrow \infty$.

We also need the following reformulation of [1], Lemma 10, which describes the connection between absence of long descending chains and short paths in the relative neighborhood graph.

Lemma 13. Let $n \geq 1$ and $b, r>0$ be such that $2 n b \leq r$. If $x, x^{\prime} \in \mathbb{R}^{2}$ are such that $\left|x-x^{\prime}\right| \leq b$ and there does not exist a $b$-bounded descending chain of more than $n$ hops starting from $x$, then $x$ and $x^{\prime}$ can be connected by a path in $\mathrm{Rng}\left(X^{(1)}\right)$ that is contained in $B_{r}(x)$. 
We show now that under the event $A_{M}^{\prime}$ shortest-path lengths in the annulus $\left(R_{M}^{-} \oplus Q_{M}(o)\right) \backslash$ $R_{M}^{-}$are not too long.

Lemma 14. If $X^{(1)} \in A_{M}^{\prime}$ and $x, x^{\prime} \in X^{(1)} \cap\left(R_{M}^{-} \oplus Q_{M / 2}(o)\right)$ are such that $\left|x-x^{\prime}\right| \leq 16 \varepsilon M$, then $x$ and $x^{\prime}$ can be connected by a path in $\operatorname{Rng}\left(X^{(1)}\right)$ of length at most $\sqrt{M}$.

Proof. Since $X^{(1)} \in A_{M}^{\prime}$, there does not exist a $16 \varepsilon M$-bounded descending chain starting from $x$ and consisting of more than $10^{4} \varepsilon^{2} M^{2}$ hops. Hence, by Lemma 13, $x$ and $x^{\prime}$ can be connected

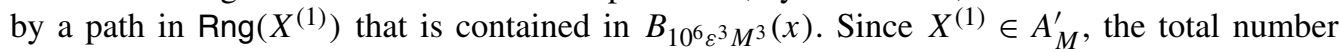

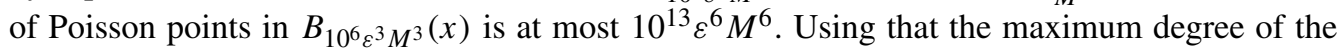
relative neighborhood graph is at most 6 , it follows that $x$ and $x^{\prime}$ can be connected by a path in $\operatorname{Rng}\left(X^{(1)}\right)$ of length at most $10^{20} \varepsilon^{9} M^{9}$. By the choice of $\varepsilon=\varepsilon(M)$, this quantity is less than $\sqrt{M}$, provided that $M$ is sufficiently large.

\subsection{The event $A_{M}^{\prime \prime}$}

The event $A_{M}^{\prime \prime}$ has to encode a certain control over shortest-path lengths on $G\left(X^{(1)}\right)$. Since it is easier to obtain a suitable amount of control when working with $G(X)$ instead of $G\left(X^{(1)}\right)$, we first introduce an auxiliary sequence of events $\left\{A_{M}^{*}\right\}_{M \geq 1}$.

To construct the events $\left\{A_{M}^{*}\right\}_{M \geq 1}$, we first collect fundamental results on Euclidean firstpassage percolation in $G(X)$. For any $P, P^{\prime} \in \mathcal{E}(X)$ recall that we let $\ell\left(P, P^{\prime}\right)=\ell_{G(X)}\left(P, P^{\prime}\right)$ denote the Euclidean length of the shortest path on $G(X)$ connecting $P$ and $P^{\prime}$. It is shown in [3] for the Delaunay triangulation and in [1] for the relative neighborhood graph that there exists a deterministic value $\mu \geq 1$, called time constant, such that almost surely

$$
\mu=\lim _{n \rightarrow \infty} n^{-1} \mathbb{E} \ell\left(o, n e_{1}\right) .
$$

If $o$ or $n e_{1}$ are not contained on $G(X)$, then we put $\ell\left(o, n e_{1}\right)=\ell\left(q(o), q\left(n e_{1}\right)\right)$, where $q(o)$ and $q\left(n e_{1}\right)$ denote the closest points of $X$ to $o$ and $n e_{1}$, respectively. In the following, we put

$$
\mu_{-}=\mu_{-}\left(\delta^{\prime}\right)=\left(1-\delta^{\prime}\right) \mu \quad \text { and } \quad \mu_{+}=\mu_{+}\left(\delta^{\prime}\right)=\left(1+\delta^{\prime}\right) \mu
$$

where $\delta^{\prime} \in(0,1)$ is a small number that will be fixed in the proof of Lemma 18 below.

Now, we construct a family of events $\left\{A_{M}^{*}\right\}_{M \geq 1}$ such that $\lim _{M \rightarrow \infty} \mathbb{P}\left(X \in A_{M}^{*}\right)=1$ and such that if $X \in A_{M}^{*}$, then the following condition is satisfied, where we consider the subsquares $Q_{\varepsilon M}\left(v_{i}\right), i \in\left\{1, \ldots, K_{M}\right\}$ introduced in the paragraph preceding Lemma 9:

(C1) Assume that $Q_{\varepsilon M}\left(v_{i}\right) \cap Q_{\varepsilon M}\left(v_{j}\right) \neq \varnothing$, i.e., the squares $Q_{\varepsilon M}\left(v_{i}\right)$ and $Q_{\varepsilon M}\left(v_{j}\right)$ are adjacent. Then, the points $q\left(v_{i}\right)$ and $q\left(v_{j}\right)$ are connected by a path $\gamma$ in $G(X)$ such that $v_{1}(\gamma) \leq \mu_{+}\left|v_{i}-v_{j}\right|$ and $\gamma$ is contained in $\left(Q_{\varepsilon M}\left(v_{i}\right) \cup Q_{\varepsilon M}\left(v_{j}\right)\right) \oplus B_{3 \varepsilon M}(o)$.

(C2) for all integers $n \geq M$ and all $P, P^{\prime} \in \mathcal{E}(X) \cap B_{2 n}(o)$ with $\left|P-P^{\prime}\right| \geq \frac{1}{2} \delta n$ it holds that (a) $P$ and $P^{\prime}$ can be connected by a path $\gamma$ in $G(X)$ such that $v_{1}(\gamma) \leq \mu_{+}\left|P-P^{\prime}\right|$ and $\gamma$ is contained in $\left[P, P^{\prime}\right] \oplus B_{n^{7 / 8} / 2}(o)$, 
(b) $\ell_{G(X)}\left(P, P^{\prime}\right) \geq \mu_{-}\left|P-P^{\prime}\right|$.

In order to show that $(\mathrm{C} 1)$ and $(\mathrm{C} 2)$ are satisfied whp, we use the following consequence of [10], Theorem 1 and Proposition 6.4.

Lemma 15. Let $E_{z, z^{\prime}}$ denote the event that there exist $P \in \mathcal{E}(X) \cap Q_{1}(z)$ and $P^{\prime} \in \mathcal{E}(X) \cap Q_{1}\left(z^{\prime}\right)$ such that

(i) $P$ and $P^{\prime}$ cannot be connected by a path $\gamma$ in $G(X)$ such that $v_{1}(\gamma) \leq \mu_{+}\left|P-P^{\prime}\right|$ and $\gamma$ is contained in $\left[P, P^{\prime}\right] \oplus B_{\left|z-z^{\prime}\right|^{7 / 8} / 8}(o)$, or

(ii) $\ell_{G(X)}\left(P, P^{\prime}\right)<\mu_{-}\left|P-P^{\prime}\right|$.

Then, $E_{z, z^{\prime}}$ decays at stretched exponential speed in $\left|z-z^{\prime}\right|$.

Lemma 16. There exists a family of events $\left\{A_{M}^{*}\right\}_{M \geq 1}$ such that $\lim _{M \rightarrow \infty} \mathbb{P}\left(X \in A_{M}^{*}\right)=1$ and for which conditions $(\mathrm{C} 1)$ and $(\mathrm{C} 2)$ are satisfied.

Proof. We say that $A_{M}^{*}$ occurs if (1) $|q(P)-P| \leq M^{1 / 64}$ for all $P \in B_{5 M}(o)$, and (2) there do not exist $z, z^{\prime} \in \mathbb{Z}^{2}$ with $\left|z-z^{\prime}\right| \geq M^{1 / 64}$ and for which $E_{z, z^{\prime}}$ occurs. By Lemma 15 , the probability of the complement of $A_{M}^{*}$ decays at stretched exponential speed in $M$.

We claim (C1) and (C2) hold under $A_{M}^{*}$. To verify condition (C1), choose $z_{i}, z_{j} \in \mathbb{Z}^{2}$ such that $q\left(v_{i}\right) \in Q_{1}\left(z_{i}\right)$ and $q\left(v_{j}\right) \in Q_{1}\left(z_{j}\right)$. Under the complement of the event $E_{z_{i}, z_{j}}$, the points $q\left(v_{i}\right)$ and $q\left(v_{j}\right)$ are connected by a path $\gamma$ in $G(X)$ such that $v_{1}(\gamma) \leq \mu_{+}\left|q\left(v_{i}\right)-q\left(v_{j}\right)\right|$ and $\gamma$ is contained in $\left[q\left(v_{i}\right), q\left(v_{j}\right)\right] \oplus B_{\left|z_{i}-z_{j}\right|^{7 / 8} / 8}(o)$. Since

$$
\left[q\left(v_{i}\right), q\left(v_{j}\right)\right] \oplus B_{\left|z_{i}-z_{j}\right|^{7 / 8} / 8}(o) \subset\left(Q_{\varepsilon M}\left(v_{i}\right) \cup Q_{\varepsilon M}\left(v_{j}\right)\right) \oplus B_{3 \varepsilon M}(o),
$$

this verifies condition (C1). To verify condition (C2), let $n \geq M$ and $P, P^{\prime} \in \mathcal{E}(X) \cap B_{2 n}(o)$ with $\left|P-P^{\prime}\right| \geq \frac{1}{2} \delta n$ be arbitrary. Furthermore, choose $z, z^{\prime} \in \overline{\mathbb{Z}}^{2}$ with $P \in Q_{1}(z)$ and $P^{\prime} \in Q_{1}\left(z^{\prime}\right)$. Under the complement of the event $E_{z, z^{\prime}}$,

(i) the points $P$ and $P^{\prime}$ can be connected by a path $\gamma$ in $G(X)$ such that $\nu_{1}(\gamma) \leq \mu_{+}\left|P-P^{\prime}\right|$ and $\gamma$ is contained in $\left[P, P^{\prime}\right] \oplus B_{n^{7 / 8} / 2}(o)$, and

(ii) $\ell_{G(X)}\left(P, P^{\prime}\right) \geq \mu_{-}\left|P-P^{\prime}\right|$,

as required.

Now, we construct a family of events $\left\{A_{M}^{\prime \prime}\right\}_{M \geq 1}$ such that $\lim _{M \rightarrow \infty} \mathbb{P}\left(X^{(1)} \in A_{M}^{\prime \prime}\right)=1$ and, almost surely, if $X^{(1)} \in A_{M}^{\prime \prime}$, then the following properties are satisfied:

(D1) Assume that $Q_{\varepsilon M}\left(v_{i}\right) \cap Q_{\varepsilon M}\left(v_{j}\right) \neq \varnothing$, i.e., the squares $Q_{\varepsilon M}\left(v_{i}\right)$ and $Q_{\varepsilon M}\left(v_{j}\right)$ are adjacent. If $Q_{\varepsilon M}\left(v_{i}\right) \cap\left(R_{M}^{-} \oplus Q_{16 \varepsilon M}(o)\right)=\varnothing$ and $Q_{\varepsilon M}\left(v_{j}\right) \cap\left(R_{M}^{-} \oplus Q_{16 \varepsilon M}(o)\right)=\varnothing$ then $\ell_{G\left(X^{(1)}\right)}\left(v_{i}, v_{j}\right) \leq \mu_{+}\left|v_{i}-v_{j}\right|$;

(D2) if $r \geq M$ and $P, P^{\prime} \in \mathcal{E}\left(X^{(1)}\right) \cap B_{r}(o)$ are such that $\left|P-P^{\prime}\right| \geq \delta r$, then the following properties are satisfied:

(a) if $\left(\left[P, P^{\prime}\right] \oplus B_{r^{7 / 8}}(o)\right) \cap R_{M}^{-}=\varnothing$, then $P$ and $P^{\prime}$ can be connected by a path $\gamma$ in $G\left(X^{(1)}\right)$ such that $\nu_{1}(\gamma) \leq \mu_{+}\left|P-P^{\prime}\right|$ and $\gamma$ is contained in $\left[P, P^{\prime}\right] \oplus B_{r^{7 / 8}}(o)$, 
(b) if $\gamma$ is any path in $G\left(X^{(1)}\right)$ connecting $P$ and $P^{\prime}$ and not hitting $R_{M}^{-} \oplus Q_{8 \varepsilon M}(o)$, then $v_{1}(\gamma) \geq \mu_{-}\left|P-P^{\prime}\right|$.

Now, we construct the family of events $\left\{A_{M}^{\prime \prime}\right\}$ announced above.

Lemma 17. There exists a family of events $\left\{A_{M}^{\prime \prime}\right\}_{M \geq 1}$ such that $\lim _{M \rightarrow \infty} \mathbb{P}\left(X^{(1)} \in A_{M}^{\prime \prime}\right)=1$ and for which conditions (D1) and (D2) are satisfied.

Proof. As in [11], Lemma 8, Fubini's theorem produces a configuration $\varphi \subset R_{M}^{-}$such that $\mathbb{P}(\varphi \cup$ $\left.X^{(1)} \in A_{M}^{*}\right) \geq \mathbb{P}\left(X \in A_{M}^{*}\right)$. Then, we let $\left\{X^{(1)} \in A_{M}^{\prime \prime}\right\}$ denote the event that $\varphi \cup X^{(1)} \in A_{M}^{*}$ and $X^{(1)} \in A_{M}^{\prime}$. In particular, Lemma 16 gives that $\lim _{M \rightarrow \infty} \mathbb{P}\left(X^{(1)} \in A_{M}^{\prime \prime}\right)=1$. Moreover, we claim that if $X^{(1)} \in A_{M}^{\prime \prime}$, then conditions (D1) and (D2) are satisfied. Regarding condition (D1) let $v_{i}$ and $v_{j}$ be subsquare centers satisfying the desired conditions. Then, by $(\mathrm{C} 1), q\left(v_{i}\right)$ and $q\left(v_{j}\right)$ are connected by a path $\gamma$ in $G\left(X^{(1)} \cup \varphi\right)$ such that $v_{1}(\gamma) \leq \mu_{+}\left|v_{i}-v_{j}\right|$ and $\gamma$ is contained in $\left(Q_{\varepsilon M}\left(v_{i}\right) \cup Q_{\varepsilon M}\left(v_{j}\right)\right) \oplus B_{3 \varepsilon M}(o)$. Since we know that $X^{(1)} \in A_{M, 1}^{\prime}$, we conclude from Lemma 9 that $\gamma$ is also a path in $G\left(X^{(1)}\right)$, so that $\ell_{G\left(X^{(1)}\right)}\left(v_{i}, v_{j}\right) \leq \mu_{+}\left|v_{i}-v_{j}\right|$. For condition (D2) we may argue similarly. Indeed, let $r \geq M$ and $P, P^{\prime} \in \mathcal{E}\left(X^{(1)}\right) \cap B_{r}(o)$ be as in condition (D2). If $\left(\left[P, P^{\prime}\right] \oplus B_{r^{7 / 8}}(o)\right) \cap R_{M}^{-}=\varnothing$, then, by condition $(\mathrm{C} 2)$ for $n=\lceil r\rceil$, the points $P, P^{\prime}$ can be connected by a path $\gamma$ in $G\left(X^{(1)} \cup \varphi\right)$ such that $\nu_{1}(\gamma) \leq \mu_{+}\left|P-P^{\prime}\right|$ and $\gamma$ is contained in $\left[P, P^{\prime}\right] \oplus B_{n^{7 / 8} / 2}(o)$. Moreover, by Lemma $9, \gamma$ is also a path in $G\left(X^{(1)}\right)$. Next, we assume that $\gamma$ is a path from $P$ to $P^{\prime}$ in $G\left(X^{(1)}\right)$ that does not hit the set $R_{M}^{-} \oplus Q_{8 \varepsilon M}(o)$. In particular, again using Lemma 9, we deduce that $\gamma$ is also a path in $G\left(X^{(1)} \cup \varphi\right)$. Therefore, condition (C2) implies that

$$
v_{1}(\gamma) \geq \ell_{G\left(X^{(1)} \cup \varphi\right)}\left(P, P^{\prime}\right) \geq \mu_{-}\left|P-P^{\prime}\right|
$$

\subsection{How $A_{M}^{\prime} \cap A_{M}^{\prime \prime} \cap E_{M}$ implies $F_{M}$}

Now, we put $A_{M}=A_{M}^{\prime} \cap A_{M}^{\prime \prime} \cap E_{M}$ and provide a key deterministic argument which shows that $X^{(1)} \in A_{M}$ implies $X^{(1)} \in F_{M}$.

Lemma 18. Let $A_{M}=A_{M}^{\prime} \cap A_{M}^{\prime \prime} \cap E_{M}$. Then $\left\{X^{(1)} \in F_{M}\right\}$ holds almost surely under the event $\left\{X^{(1)} \in A_{M}\right\}$.

As noted in the beginning of this section, once Lemma 18 is established, the proof of Proposition 5 is complete.

To prove Lemma 18, we proceed by contradiction. Hence, using Lemma 8, we assume that there exists $z \in 4 \mathbb{Z} \times 2 \mathbb{Z}, x^{-} \in X^{(1)} \cap\left(M z+H^{-}\right)$and $x^{+} \in X^{(1)} \cap\left(M z+H^{+}\right)$such that

(i) $n=\pi_{1}(z)<0$ and $\left[x^{-}, x^{+}\right]$forms an edge in $G\left(X^{(1)}\right)$ that is contained in $B_{M / 2}(M z)$,

(ii) $\gamma=\gamma_{x^{+}}^{(n M)}$ exists, $\gamma[P]$ coalesces with $\gamma_{\mathrm{m}}$ and $\left|\pi_{2}(P)\right| \leq \delta M$, where $P$ denotes the last intersection point of $\gamma$ and $l_{0}^{\mathrm{v}}$.

Note that the reduction to the case $n<0$ is a consequence of the remark after Lemma 8 . 


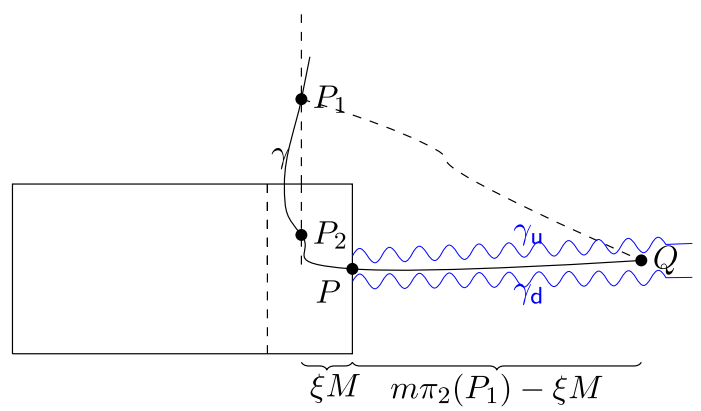

Figure 5. Illustration of the event $A_{M}^{(1)}$.

Now, we distinguish several cases. First, assume that there exists $\xi \in[0,1-\delta]$ such that $\gamma$ intersects the union of rays $\{-\xi M\} \times(\mathbb{R} \backslash[-M, M])$ at some point $P_{1}$. Without loss of generality, we assume that $\pi_{2}\left(P_{1}\right) \geq M$ and that $P_{1}$ is the last such intersection point. Furthermore, let $Q$ denote a point of $\gamma[P]$ satisfying $\pi_{1}(Q)=m \pi_{2}\left(P_{1}\right)-\xi M$. Here, $m \geq 2$ is a sufficiently large integer that will be fixed in the course of the proof. Figure 5 provides a rough illustration. In particular, it does not show edges crossing $R_{M}^{-}$.

Let $A_{M}^{(1)}$ denote the event that $A_{M} \cap F_{M}^{c}$ occurs and that a point $P_{1}$ as described above exists. Under the event $A_{M}^{(1)}$ we derive upper and lower bounds on the shortest-path length $\ell^{(-4 M)}\left(P_{1}, Q\right)$ that cannot be satisfied simultaneously.

Lemma 19. Almost surely under the event $A_{M}^{(1)}$,

$$
\ell^{(-4 M)}\left(P_{1}, Q\right) \leq \mu_{+} \pi_{2}\left(P_{1}\right)\left(\left(m+m^{-1}\right)+3 \delta m\right) .
$$

Lemma 20. Almost surely under the event $A_{M}^{(1)}$,

$$
\ell^{(n M)}(P, Q) \geq \mu_{-}\left(m \pi_{2}\left(P_{1}\right)-\xi M\right) .
$$

In order to derive lower bounds for $\ell^{(n M)}\left(P_{1}, P\right)$, we need to further decompose the event $A_{M}^{(1)}$. More precisely, let $A_{M}^{(1, a)}$ denote the event that $A_{M}^{(1)}$ occurs and that $\gamma\left[P_{1}\right]$ stays within the vertical half-plane $[-\xi M, \infty) \times \mathbb{R}$. Then, we have the following lower bounds for $\ell^{(n M)}\left(P_{1}, P\right)$.

Lemma 21. Almost surely under the event $A_{M}^{(1, a)}$,

$$
\ell^{(n M)}\left(P_{1}, P\right) \geq \mu_{-}\left((\sqrt{2}-1) \pi_{2}\left(P_{1}\right)+(\xi-\delta) M\right) .
$$

Conversely, we put $A_{M}^{(1, b)}=A_{M}^{(1)} \backslash A_{M}^{(1, a)}$ and observe that under $A_{M}^{(1, b)}$ the path $\gamma\left[P_{1}, P\right]$ intersects the segment $\{-\xi M\} \times[-M, M]$. We let $P_{2}=(-\xi M, \eta M)$ denote the last such intersection point. 
Lemma 22. Almost surely under the event $A_{M}^{(1, b)}$,

$$
\ell^{(n M)}\left(P_{1}, P\right) \geq \mu_{-}\left(\pi_{2}\left(P_{1}\right)+(\xi-2) M+\sqrt{1+\eta^{2}} M-2 \delta M\right)+(1-\eta) M .
$$

Before we provide proofs of Lemmas 19-22, we show to deduce from them a contradiction.

Lemma 23. There exists $m_{0} \geq 1$ such that $\mathbb{P}\left(A_{M}^{(1)}\right)=0$ holds for all $m \geq m_{0}$, all sufficiently small $\delta, \delta^{\prime} \in(0,1)$ and all sufficiently large $M \geq 1$.

Proof. We start by showing $\mathbb{P}\left(A_{M}^{(1, a)}\right)=0$. First, we recall that $\gamma$ is a geodesic in $G\left(X^{(1)}\right)$ with respect to $v_{1}^{(n M)}$, so that

$$
\ell^{(-4 M)}\left(P_{1}, Q\right) \geq \ell^{(n M)}\left(P_{1}, Q\right)=\ell^{(n M)}\left(P_{1}, P\right)+\ell^{(n M)}(P, Q) .
$$

Hence, Lemmas 19, 20 and 21 give that

$$
\mu_{-} \pi_{2}\left(P_{1}\right)(m+\sqrt{2}-1-\delta m) \leq \mu_{+} \pi_{2}\left(P_{1}\right)\left(\left(m+m^{-1}\right)+3 \delta m\right),
$$

so that

$$
\mu_{-}(\sqrt{2}-1) \leq m\left(4 \mu_{+} \delta+\mu_{+}-\mu_{-}\right)+m^{-1} \mu_{+} .
$$

Since $\sqrt{2}>1$ this yields a contradiction if first $m \geq 1$ is chosen sufficiently large and then $\delta, \delta^{\prime} \in(0,1)$ are chosen sufficiently small.

To show $\mathbb{P}\left(A_{M}^{(1, b)}\right)=0$, we proceed similarly. Using Lemmas 19, 20 and 22, we obtain that

$$
\mu_{-}\left((m+1) \pi_{2}\left(P_{1}\right)-2 M+\sqrt{1+\eta^{2}} M\right)+(1-\eta) M \leq \mu_{+} \pi_{2}\left(P_{1}\right)\left(\left(m+m^{-1}\right)+5 \delta m\right),
$$

which gives that

$$
5 \mu_{+} \delta m+\mu_{+}\left(m+m^{-1}\right)-\mu_{-} m \geq \mu_{-}+\frac{M \mu_{-}}{\pi_{2}\left(P_{1}\right)}\left(\frac{1-\eta}{\mu_{-}}+\sqrt{1+\eta^{2}}-2\right) .
$$

First, the left-hand side becomes arbitrarily small if first $m \geq 1$ is chosen sufficiently large and then $\delta, \delta^{\prime} \in(0,1)$ are chosen sufficiently small. Moreover, the right-hand side is bounded below by

$$
\mu_{-}\left(1-\frac{M}{\pi_{2}\left(P_{1}\right)}\left(1-\frac{1-\eta}{\mu_{-}}-\sqrt{1+\eta^{2}}+1\right)\right) \geq \mu_{-} \min \left\{1, \frac{1-\eta}{\mu_{-}}+\sqrt{1+\eta^{2}}-1\right\} .
$$

Now a quick computation shows that the right-hand side is bounded below by $1 / 4$, which gives the desired contradiction.

Now, we prove Lemmas 19-22.

Proof of Lemma 19. First, by uniqueness of $\hat{u}_{0}$-geodesics in $G\left(X^{(1)}\right)$ with respect to $v_{1}^{(0)}$, the geodesic $\gamma[P]$ is trapped between $\gamma_{\mathrm{u}}$ and $\gamma_{\mathrm{d}}$. Writing $P_{\mathrm{u}}^{\prime}, P_{\mathrm{d}}^{\prime}$ for the extremal intersection points 
of the vertical line $l_{m \pi_{2}\left(P_{1}\right)-\xi M}^{\mathrm{v}}$ with the dilated cones $C_{\delta}\left(P_{\mathrm{u}}\right) \oplus B_{\delta M}(o)$ and $C_{\delta}\left(P_{\mathrm{d}}\right) \oplus B_{\delta M}(o)$, the cone condition (iii) in the event $E_{M}$ implies that

$$
\left|\pi_{2}(Q)\right| \leq \max \left\{\left|\pi_{2}\left(P_{\mathrm{u}}^{\prime}\right)\right|,\left|\pi_{2}\left(P_{\mathrm{d}}^{\prime}\right)\right|\right\}+2 \delta M .
$$

In particular, using the location of the points $P_{\mathrm{u}}, P_{\mathrm{d}}$ in the event $E_{M}$, we conclude that for small $\delta$,

$$
\left|\pi_{2}(Q)\right| \leq \max \left\{\left|\pi_{2}\left(P_{\mathrm{u}}\right)\right|,\left|\pi_{2}\left(P_{\mathrm{d}}\right)\right|\right\}+m \pi_{2}\left(P_{1}\right) \tan (2 \delta)+2 \delta M \leq 3 \delta m \pi_{2}\left(P_{1}\right) .
$$

Next, we apply condition (D2)(a) with $r=2 m \pi_{2}\left(P_{1}\right), P=P_{1}$ and $P^{\prime}=Q$. For this, we need to verify that

$$
\left(\left[P_{1}, Q\right] \oplus B_{\left.\left(2 m \pi_{2}\left(P_{1}\right)\right)^{7 / 8}(o)\right) \cap R_{M}^{-}=\varnothing .}\right.
$$

If $\pi_{2}\left(P_{1}\right) \leq 5 M$, then the distance of the half-plane $[(-1+\delta) M, \infty) \times \mathbb{R}$ to $R_{M}^{-}$is larger than $\left(2 m \pi_{2}\left(P_{1}\right)\right)^{7 / 8}$. Hence, we may assume that $\pi_{2}\left(P_{1}\right)>5 M$. Now, let $P^{\prime \prime} \in\left[P_{1}, Q\right]$ be arbitrary. If $\pi_{1}\left(P^{\prime \prime}\right) \leq \pi_{2}\left(P_{1}\right)$, then $\pi_{2}\left(P^{\prime \prime}\right) \geq \frac{1}{2} \pi_{2}\left(P_{1}\right)$, so that the distance between $P^{\prime \prime}$ and $R_{M}^{-}$is larger than $\frac{1}{4} \pi_{2}\left(P_{1}\right) \geq\left(2 m \pi_{2}\left(P_{1}\right)\right)^{7 / 8}$. Otherwise, $\pi_{1}\left(P^{\prime \prime}\right) \geq \pi_{2}\left(P_{1}\right)$, which implies that the distance between $P^{\prime \prime}$ and $R_{M}^{-}$is larger than $\pi_{2}\left(P_{1}\right) \geq\left(2 m \pi_{2}\left(P_{1}\right)\right)^{7 / 8}$.

Since the path provided by condition (D2)(a) is contained in the half-plane $[-4 M, \infty) \times \mathbb{R}$, we obtain the upper bound $\ell^{(-4 M)}\left(P_{1}, Q\right) \leq \mu_{+}\left|P_{1}-Q\right|$. Therefore,

$$
\begin{aligned}
\ell^{(-4 M)}\left(P_{1}, Q\right) & \leq \mu_{+}\left(\sqrt{m^{2}+1} \pi_{2}\left(P_{1}\right)+\left|\pi_{2}(Q)\right|\right) \\
& \leq \mu_{+}\left(m \pi_{2}\left(P_{1}\right)+\frac{1}{m+\sqrt{m^{2}+1}} \pi_{2}\left(P_{1}\right)+\left|\pi_{2}(Q)\right|\right) \\
& \leq \mu_{+}\left(\left(m+m^{-1}\right) \pi_{2}\left(P_{1}\right)+\left|\pi_{2}(Q)\right|\right) .
\end{aligned}
$$

In particular,

$$
\ell^{(-4 M)}\left(P_{1}, Q\right) \leq \mu_{+}\left(m+m^{-1}\right) \pi_{2}\left(P_{1}\right)+3 \delta m \mu_{+} \pi_{2}\left(P_{1}\right)
$$

as required.

Proof of Lemma 20. By the choice of $P$, the geodesic $\gamma[P]$ does not intersect $R_{M}^{-} \oplus Q_{8 \varepsilon M}(o)$. Hence, condition (D2)(b) gives that $\ell^{(n M)}(P, Q)=v_{1}(\gamma[P, Q])$ is bounded from below by

$$
\mu_{-}|P-Q| \geq \mu_{-}\left(\pi_{1}(Q)-\pi_{1}(P)\right) \geq \mu_{-}\left(m \pi_{2}\left(P_{1}\right)-\xi M\right),
$$

as required.

Proof of Lemma 21. Since $\gamma\left[P_{1}\right]$ is contained in $[-\xi M, \infty) \times \mathbb{R}$, condition (D2)(b) shows that

$$
\begin{aligned}
\ell^{(n M)}\left(P_{1}, P\right) & \geq \mu_{-}\left(\left|P_{1}\right|-|P|\right) \geq \mu_{-}\left(\sqrt{\pi_{2}\left(P_{1}\right)^{2}+\xi^{2} M^{2}}-\delta M\right) \\
& \geq \mu_{-}\left((\sqrt{2}-1) \pi_{2}\left(P_{1}\right)+(\xi-\delta) M\right),
\end{aligned}
$$

where the last inequality holds since the function $x \mapsto \sqrt{\pi_{2}\left(P_{1}\right)^{2}+x^{2}}-x$ is decreasing. 


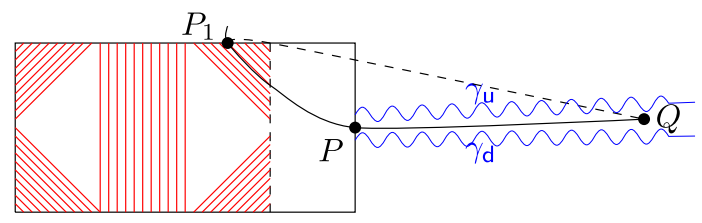

Figure 6. Illustration of the second case.

Proof of Lemma 22. Let $P_{2}^{\prime}$ denote the first point on $\gamma\left[P_{1}, P\right]$ with $\pi_{2}\left(P_{2}^{\prime}\right)=(1+\delta) M$. If $\pi_{2}\left(P_{1}\right) \leq(1+\delta) M$, we put $P_{2}^{\prime}=P_{1}$. Now, by condition (D2)(b),

$$
\ell^{(n M)}\left(P_{1}, P_{2}\right) \geq \mu_{-}\left|P_{1}-P_{2}^{\prime}\right|+\left|P_{2}^{\prime}-P_{2}\right| \geq \mu_{-}\left(\pi_{2}\left(P_{1}\right)-M-\delta M\right)+(1-\eta) M .
$$

Similarly,

$$
\ell^{(n M)}\left(P_{2}, P\right) \geq \mu_{-}\left|P_{2}-P\right| \geq \mu_{-}\left(\left|P_{2}\right|-\delta M\right) .
$$

In particular, combining the lower bounds above yields that

$$
\begin{aligned}
\ell^{(n M)}\left(P_{1}, P\right) & \geq \mu_{-}\left(\pi_{2}\left(P_{1}\right)-M+\sqrt{\xi^{2}+\eta^{2}} M-2 \delta M\right)+(1-\eta) M \\
& \geq \mu_{-}\left(\pi_{2}\left(P_{1}\right)+(\xi-2) M+\sqrt{1+\eta^{2}} M-2 \delta M\right)+(1-\eta) M,
\end{aligned}
$$

as required.

Hence, in the following we may assume that $\gamma$ does not hit the area $[(-1+\delta) M, 0] \times(\mathbb{R} \backslash$ $[-M, M])$. Recall that there is no left-right crossing in $R_{M}^{-}$, see Figure 3. Therefore, $\gamma$ crosses the union of segments $[(-2-\delta) M,(-1+\delta) M] \times\{ \pm M\}$. We let $P_{1}=\left(-\xi M, \pi_{2}\left(P_{1}\right)\right)$ denote the last such intersection point and assume that $\pi_{2}\left(P_{1}\right)=M$. Next, we let $Q$ denote a point on $\gamma[P]$ satisfying $\pi_{1}(Q)=(m-1) M$, where $m \geq 3$ is again a sufficiently large integer, see Figure 6.

The proof of Lemma 18 in the current setting is similar to what we have seen above, but for the convenience of the reader, we include some details. More precisely, putting $A_{M}^{(2)}=A_{M} \cap F_{M}^{c} \backslash$ $A_{M}^{(1)}$ we derive contradictory upper and lower bounds on the shortest-path length $\ell^{(-4 M)}\left(P_{1}, Q\right)$.

Lemma 24. Almost surely under the event $A_{M}^{(2)}$ it holds that

$$
\ell^{(-4 M)}\left(P_{1}, Q\right) \leq \mu_{+}\left(m+m^{-1}+\xi-1+6 \delta m\right) M .
$$

To derive the lower bounds we introduce a further auxiliary point $P_{2}=\left(\pi_{1}\left(P_{2}\right), \eta M\right)$ as last point of $\gamma\left[P_{1}\right]$ satisfying $\pi_{1}\left(P_{2}\right)=-(1-\delta) M$. First, we provide lower bounds for $\ell^{(n M)}\left(P_{1}, P_{2}\right)$ and $\ell^{(n M)}\left(P_{2}, Q\right)$. 
Lemma 25. Almost surely under the event $A_{M}^{(2)}$ it holds that

$$
\ell^{(n M)}\left(P_{1}, P_{2}\right) \geq(1-\eta) M
$$

and

$$
\ell^{(n M)}\left(P_{2}, Q\right) \geq \mu_{-}\left(m-1+\sqrt{\eta^{2}+1}-2 \delta m\right) M .
$$

If we let $A_{M}^{(2, a)}$ denote the event that $A_{M}^{(2)}$ occurs and $\xi \leq 1+4 \delta$, then the lower bounds derived in Lemma 25 are sufficient to arrive at the desired contradiction. However, if the event $A_{M}^{(2, b)}=A_{M}^{(2)} \backslash A_{M}^{(2, a)}$ occurs, then a more refined reasoning is necessary. By Remark 4 this case cannot occur if $G\left(X^{(1)}\right)=\operatorname{Rng}\left(X^{(1)}\right)$, so that we may assume $G\left(X^{(1)}\right)=\operatorname{Del}\left(X^{(1)}\right)$.

Lemma 26. Almost surely under the event $A_{M}^{(2, b)}$ it holds that

$$
\ell^{(n M)}\left(P_{1}, P_{2}\right) \geq(\sqrt{2}(\xi-1)+|2-\xi-\eta|-2 \delta) M .
$$

Before we provide proofs of Lemmas 24-26, we show how to deduce from them a contradiction.

Lemma 27. There exists $m_{0}^{\prime} \geq 1$ such that $\mathbb{P}\left(A_{M}^{(2)}\right)=0$ for all $m \geq m_{0}^{\prime}$, all sufficiently small $\delta, \delta^{\prime} \in(0,1)$ and all sufficiently large $M \geq 1$.

Proof. We start by showing $\mathbb{P}\left(A_{M}^{(2, a)}\right)=0$. Under the event $A_{M}^{(2, a)}$ we have $\xi \leq 1+4 \delta$, so that Lemmas 24 and 25 give that

$$
\mu_{+}\left(m+m^{-1}+10 \delta m\right) \geq 1-\eta+\mu_{-}\left(m-1+\sqrt{\eta^{2}+1}-2 \delta m\right) .
$$

Hence,

$$
m\left(\mu_{+}-\mu_{-}\right)+\mu_{+}\left(m^{-1}+12 \delta m\right) \geq 1-\eta+\mu_{-}\left(\sqrt{\eta^{2}+1}-1\right) \geq \frac{1}{4},
$$

as required.

Next, we prove $\mathbb{P}\left(A_{M}^{(2, b)}\right)=0$. Again, by Lemmas 24,25 and 26 shows that

$$
\mu_{+}\left(m+\xi-1+m^{-1}+10 m \delta\right) \geq \mu_{-}\left(m-1+\sqrt{\eta^{2}+1}\right)+\sqrt{2}(\xi-1)+|2-\xi-\eta| .
$$

After rearranging terms, we arrive at

$$
\begin{aligned}
& m\left(\mu_{+} \mu_{-}^{-1}-1\right)+\mu_{+} \mu_{-}^{-1}\left(m^{-1}+10 m \delta\right) \\
& \quad \geq-1+\sqrt{\eta^{2}+1}+\frac{\sqrt{2}-\mu_{+}}{\mu_{-}}(\xi-1)+\frac{1}{\mu_{-}}|2-\xi-\eta| .
\end{aligned}
$$


First, the left-hand side becomes arbitrarily small if first $m$ is chosen sufficiently large and then $\delta, \delta^{\prime} \in(0,1)$ are chosen sufficiently small. Next, it is shown in [4] that $\mu \leq \frac{35}{3 \pi^{2}}<\sqrt{2}$ holds in the Delaunay case, so that the coefficient $\left(\sqrt{2}-\mu_{+}\right) / \mu_{-}$is bounded away from 0 . Now, we can distinguish between the three cases (i) $|\eta| \geq 1 / 4$ (ii) $\xi \geq 5 / 4$ and (iii) $|\eta| \leq 1 / 4$ and $\xi \leq 5 / 4$ to see that the right-hand side remains bounded away from 0 . More precisely, if $|\eta| \geq 1 / 4$, then

$$
-1+\sqrt{\eta^{2}+1}=\frac{\eta^{2}}{1+\sqrt{\eta^{2}+1}} \geq \frac{1}{48} .
$$

If $\xi \geq 5 / 4$, then $\frac{\sqrt{2}-\mu_{+}}{\mu_{-}}(\xi-1) \geq \frac{\sqrt{2}-\mu_{+}}{4 \mu_{-}}$. Finally, if $|\eta| \leq 1 / 4$ and $\xi \leq 5 / 4$, then

$$
\frac{1}{\mu_{-}}|2-\xi-\eta| \geq \frac{1}{2 \mu_{-}},
$$

as required.

Finally, we provide the proofs of Lemmas 24-26.

Proof of Lemma 24. Choose a subsquare center $v_{i}$ such that $\pi_{2}\left(v_{i}\right)=M+9.5 \varepsilon M$ and $\mid \pi_{1}\left(v_{i}\right)+$ $\xi M \mid \leq \varepsilon M$. Then, by Lemma 14 , we obtain that $\ell^{(-4 M)}\left(P_{1}, P_{1}^{\prime}\right) \leq \sqrt{M}$, where $P_{1}^{\prime}=q\left(v_{i}\right)$. Next, choose a subsquare center $v_{j}$ such that $\pi_{2}\left(v_{j}\right)=\pi_{2}\left(v_{i}\right)$ and

$$
\left|\pi_{1}\left(v_{j}\right)-(-1+\delta) M\right| \leq \varepsilon M .
$$

Next, putting $P_{3}=q\left(v_{j}\right)$, we leverage (D1) to bound $\ell^{(-4 M)}\left(P_{1}^{\prime}, P_{3}\right)$. Although $Q_{\varepsilon} M\left(v_{i}\right)$ and $Q_{\varepsilon}\left(v_{j}\right)$ are not adjacent, they are connected through a sequence of adjacent squares, so that we can apply condition (D1) to each of the adjacent pairs. For each such pair, this gives us a connecting path whose length is of order $\varepsilon M$ and which is therefore contained in the half-plane $[-4 M, \infty) \times \mathbb{R}$. Combining the upper bounds for connection lengths between adjacent squares, we therefore arrive at $\ell^{(-4 M)}\left(P_{1}^{\prime}, P_{3}\right) \leq \mu_{+}(\xi-1+\delta+2 \varepsilon) M$. Finally, noting that

$$
\begin{aligned}
\left|P_{3}-Q\right| & \leq \sqrt{\left(\pi_{1}\left(P_{3}\right)-\pi_{1}(Q)\right)^{2}+\pi_{2}\left(P_{3}\right)^{2}}+\left|\pi_{2}(Q)\right| \\
& \leq \sqrt{m^{2}+(1+\delta)} M+3 \delta m M \\
& \leq \sqrt{m^{2}+1}+4 \delta m M,
\end{aligned}
$$

an application of (D2)(a) results in

$$
\ell^{(-4 M)}\left(P_{3}, Q\right) \leq \mu_{+}\left(\sqrt{m^{2}+1}+4 \delta m\right) M \leq \mu_{+}\left(m+m^{-1}+4 \delta m\right) M,
$$

as required.

Proof of Lemma 25. First, note that $\eta \leq 1$ as $\gamma$ does not hit the set $[-(1-\delta) M, 0] \times(\mathbb{R} \backslash$ $[-M, M])$. In particular, $\ell^{(n M)}\left(P_{1}, P_{2}\right) \geq(1-\eta) M$, which proves the first claim. 
Second, proceeding as in Lemma 20, condition (D2)(b) implies that

$$
\ell^{(n M)}\left(P_{2}, P\right) \geq \mu_{-}\left(\sqrt{\eta^{2}+1}-2 \delta\right) M
$$

and $\ell^{(n M)}(P, Q) \geq \mu_{-}(m-1) M$. Hence,

$$
\ell^{(n M)}\left(P_{2}, Q\right)=\ell^{(n M)}\left(P_{2}, P\right)+\ell^{(n M)}(P, Q) \geq \mu_{-}\left(m-1+\sqrt{\eta^{2}+1}-2 \delta m\right) M,
$$

as required.

Proof of Lemma 26. First, Corollary 11 and Remark 3 show that the first edge in $\gamma\left[P_{1}\right]$ is one of the diagonal edges shown in Figure 3 . Hence by Corollary 11, writing $P_{1}^{\prime}$ for intersection point of this edge with the vertical line $l_{-M}^{\mathrm{v}}$, we arrive at

$$
\ell^{(n M)}\left(P_{1}, P_{2}\right) \geq \ell^{(n M)}\left(P_{1}, P_{1}^{\prime}\right)+\ell^{(n M)}\left(P_{1}^{\prime}, P_{2}\right) \geq \sqrt{2}(\xi-1) M+\left|\pi_{2}\left(P_{1}^{\prime}\right)-\pi_{2}\left(P_{2}\right)\right|-\delta M .
$$

Since the right-hand side is at least $(\sqrt{2}(\xi-1)+|2-\xi-\eta|-2 \delta) M$, we conclude the proof.

\section{Proof of Theorem 2}

The proof of Theorem 2 follows the method developed initially in [2] for the Radial Spanning Tree and thus generalized in [5] to other geometric random trees. The proof below, and especially Step 1, is very close to the one given in Section 5 of [5] about the Euclidean FPP Trees. In order to make the paper self-contained, we recall the main steps of the method and insist on the new parts which are proper to the context $G(X)=\operatorname{Del}(X)$ or $\operatorname{Rng}(X)$.

To begin with, we provide an overview for the proof of Theorem 2. Let $\hat{u} \in S^{1}$ be a given direction. By isotropy, it suffices to prove that the expectation of $\chi_{r}(\hat{u}, 2 \pi)$ tends to 0 as $r$ tends to infinity. The proof works as well when $2 \pi$ is replaced with any $c>0$.

The spirit of the proof of Theorem 2 is the following. First, a uniform moment condition reduces the proof to the convergence in probability of $\chi_{r}(\hat{u}, 2 \pi)$ to 0 . Thus, far away from the origin, the radial character of the geodesics of tree $\mathcal{T}_{x^{\star}}$ vanishes. In other words, when $r$ is large, with high probability, the shortest-path tree $\mathcal{T}_{x^{\star}}$ locally looks like a directed forest around $r \hat{u}$. This directed forest is in fact made up of all the semi-infinite geodesics $\gamma_{x}$ with direction $-\hat{u}$ starting from all the Poisson points $x \in X$. Let us denote this forest by $\mathcal{F}_{-\hat{u}}$. Henceforth, to a semi-infinite geodesic in $\mathcal{T}_{x^{\star}}$ crossing the arc of $S_{r}(o)$ centered at $r \hat{u}$ and with length $2 \pi$, it corresponds a bi-infinite geodesic in the directed forest $\mathcal{F}_{-\hat{u}}$. Now, Theorem 1 implies that this event should not occur.

In the sequel, the geodesic $\gamma$ will refer to a sequence of vertices $\left(x_{1}, \ldots, x_{n}\right)$ as well as to the union of segments $\left[x_{i}, x_{i+1}\right]$ for $i=1, \ldots, n-1$.

Step 1: A classical use of Fubini's theorem - see [11], Lemma 6, [15], Theorem 0, or Lemma 3 above - ensures the existence with probability 1 of exactly one semi-infinite geodesic, say $\gamma_{x}$, with direction $-\hat{u}$ starting from every Poisson point $x \in X$. The collection of these semi-infinite 
geodesics provides a directed forest $\mathcal{F}_{-\hat{u}}$. The goal of this first step is to approximate the shortestpath tree $\mathcal{T}_{x^{\star}}$ inside the disk $B_{L}(r \hat{u})$ by the directed forest $\mathcal{F}_{-\hat{u}}$ : see (3) below.

Let us first remark that $\mathcal{T}_{x^{\star}}$ and $\mathcal{F}_{-\hat{u}}$ can be viewed as outdegree 1 graphs (except for the root $x^{\star}$ of $\mathcal{T}_{x^{\star}}$ ). Indeed, the outgoing vertex of any $x \in X$ is defined as its (a.s. only) neighbour belonging to $\gamma_{x^{\star}, x}$ for $\mathcal{T}_{x^{\star}}$, or to $\gamma_{x}$ for $\mathcal{F}_{-\hat{u}}$. This interpretation provides the tree $\mathcal{T}_{x^{\star}}$ with a direction, from infinity to $x^{\star}$.

Moreover, both graphs $\mathcal{T}_{x^{\star}}$ and $\mathcal{F}_{-\hat{u}}$ can be built simultaneously on the same vertex set $X$. A measurable function $F$ is said local if there exists a deterministic real number $L>0$ such that, for any $z \in \mathbb{R}^{2}$, the quantities $F\left(z, \mathcal{T}_{x^{\star}}\right)$ and $F\left(z, \mathcal{F}_{-\hat{u}}\right)$ are equal whenever each Poisson point $x \in X \cap B_{L}(z)$ admits the same outgoing vertex in $\mathcal{T}_{x^{\star}}$ and $\mathcal{F}_{-\hat{u}}$. Our local approximation result is written through the use of local functions: for any local function $F$,

$$
\lim _{r \rightarrow \infty} d_{\mathrm{TV}}\left(F\left(r \hat{u}, \mathcal{T}_{x^{\star}}\right), F\left(o, \mathcal{F}_{-\hat{u}}\right)\right)=0,
$$

where $d_{\mathrm{TV}}$ denotes the total variation distance.

Let $F$ be a local function with local parameter $L$. Then, by translation invariance of the directed forest $\mathcal{F}_{-\hat{u}}$,

$$
\begin{aligned}
\mathcal{A}(r) & :=d_{\operatorname{TV}}\left(F\left(r \hat{u}, \mathcal{T}_{x^{\star}}\right), F\left(o, \mathcal{F}_{-\hat{u}}\right)\right)=d_{\operatorname{TV}}\left(F\left(r \hat{u}, \mathcal{T}_{x^{\star}}\right), F\left(r \hat{u}, \mathcal{F}_{-\hat{u}}\right)\right) \\
& \leq \mathbb{P}\left(F\left(r \hat{u}, \mathcal{T}_{x^{\star}}\right) \neq F\left(r \hat{u}, \mathcal{F}_{-\hat{u}}\right)\right) .
\end{aligned}
$$

The event $F\left(r \hat{u}, \mathcal{T}_{x^{\star}}\right) \neq F\left(r \hat{u}, \mathcal{F}_{-\hat{u}}\right)$ implies the existence of a Poisson point $x$ in $B_{L}(r \hat{u})$ whose outgoing vertices in $\mathcal{T}_{x^{\star}}$ and $\mathcal{F}_{-\hat{u}}$ are different. So, by uniqueness, the geodesics $\gamma_{x^{\star}, x}$ (in $\mathcal{T}_{x^{\star}}$ ) and $\gamma_{x}$ (in $\mathcal{F}_{-\hat{u}}$ ) have only $x$ in common. Hence, for $\varepsilon \in(0,1)$,

$$
\mathcal{A}(r) \leq \mathbb{P}\left(\exists x \in X \cap B_{L}(r \hat{u}) \text { s.t. } \gamma_{x^{\star}, x} \cap \gamma_{x}=\{x\} \text { and } x^{\star} \in B_{r^{\varepsilon}}(o)\right)+o(1) .
$$

Thus, because of translation invariance and the identity $\gamma_{x^{\star}, x}=\gamma_{x, x^{\star}}$, we get

$$
\mathcal{A}(r) \leq \mathbb{P}\left(\exists x \in X \cap B_{L}(o), \exists x^{\prime} \in X \cap B_{r^{\varepsilon}}(-r \hat{u}) \text { s.t. } \gamma_{x, x^{\prime}} \cap \gamma_{x}=\{x\}\right)+o(1) .
$$

The event in the r.h.s. of (5) is described in Figure 7.

Besides, we can require that the geodesics $\gamma_{x}$ and $\gamma_{x, x^{\prime}}$ belong to a thin cone with direction $-r \hat{u}$. For $\eta>0$ and $z \in \mathbb{R}^{2}$, let $C(z, \eta)$ be the cone with apex $o$, direction the vector $z$ and opening angle $\eta: C(z, \eta)=\left\{z^{\prime} \in \mathbb{R}^{2}, \theta\left(z, z^{\prime}\right) \leq \eta\right\}$ where $\theta\left(z, z^{\prime}\right)$ is the absolute value of the angle (in $[0 ; \pi])$ between vectors $z$ and $z^{\prime}$. On the one hand, the semi-infinite geodesic $\gamma_{x}$ has asymptotic direction $-\hat{u}$. So, with high probability, for any $\eta>0$ and $M$ large enough, its restriction to the outside of the disk $B_{M}(o)$ is included in the cone $C(-\hat{u}, \eta)$. See [5], Lemma 13, for details. On the other hand, the same statement holds for the geodesic $\gamma_{x, x^{\prime}}$ using the moderate deviations result [10], Theorem 2.2. See [5], Lemma 14, for details. Consequently, for any $\eta>0$ and $M, r$ large enough, we have $\mathcal{A}(r) \leq \mathbb{P}\left(A_{r}\right)+o(1)$, where the event $A_{r}$ is defined by: there exist $x \in X \cap$ $B_{L}(o)$ and $x^{\prime} \in X \cap B_{r^{\varepsilon}}(-r \hat{u})$ such that $\gamma_{x, x^{\prime}} \cap \gamma_{x}=\{x\}$ and $\left(\gamma_{x, x^{\prime}} \cup \gamma_{x}\right) \cap B_{M}(o)^{c} \subset C(-\hat{u}, \eta)$. On the event $A_{r}$, the shortest-path tree $\mathcal{T}_{x}$ contains two disjoint (except the root $x$ ) geodesics with direction $-\hat{u}$ which are as long as we want. However, with probability $1, \mathcal{T}_{x}$ contains at most one 


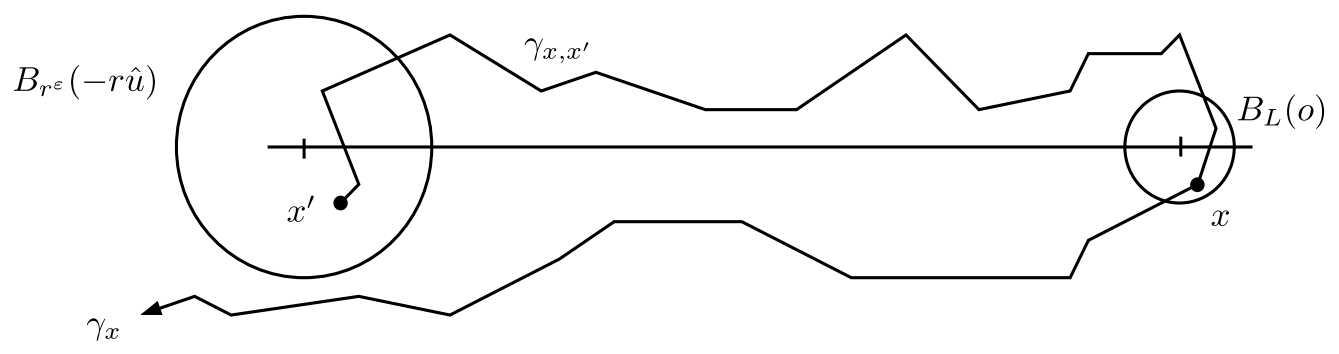

Figure 7. This picture represents the event appearing in (5) with $\hat{u}=(1,0)$. Poisson points $x$ and $x^{\prime}$ respectively belong to the disks $B_{L}(o)$ and $B_{r} \varepsilon(-r \hat{u})$. The shortest-path tree $\mathcal{T}_{x}$ contains a semi-infinite geodesic $\gamma_{x}$ with asymptotic direction $-\hat{u}$ and a geodesic $\gamma_{x, x^{\prime}}$ whose endpoint is in $B_{r^{\varepsilon}}(-r \hat{u})$. Furthermore, $\gamma_{x}$ and $\gamma_{x, x^{\prime}}$ have only the vertex $x$ in common.

semi-infinite geodesic with (deterministic) asymptotic direction $-\hat{u}$. So $\mathbb{P}\left(A_{r}\right)$ is a $o(1)$ which leads to (3)

Step 2: The goal of this second step is to state that the directed forest $\mathcal{F}_{-\hat{u}}$ does not contain any bi-infinite geodesic with probability 1 . This is a consequence of coalescence of semi-infinite geodesics (i.e., Theorem 1).

Let $\hat{v} \in S^{1}$ be orthogonal to $\hat{u}$. Let $\ell$ be the line spanned by the vector $\hat{v}$ and, for any $m>0$, let $\ell_{m}=\ell-m \hat{u}$. For $x<y$, we also denote by $\ell_{m}(x, y)$ the subset of $\ell_{m}$ defined by

$$
\ell_{m}(x, y)=\left\{-m \hat{u}+b \hat{v} \in \mathbb{R}^{2} ; x \leq b<y\right\} .
$$

Thus, we denote by $K\left[\ell_{0}(x, y)\right]$ the number of elements $P \in \ell_{0}(x, y)$ which are defined as the last intersection point between a bi-infinite geodesic of the directed forest $\mathcal{F}_{-\hat{u}}$ and the line $\ell_{0}$. In the same way, we denote by $K\left[\ell_{0}(x, y), \ell_{m}\right]$ the number of elements $P \in \ell_{m}$ which are defined as the last intersection point between a bi-infinite geodesic $\gamma$ of $\mathcal{F}_{-\hat{u}}$ and the line $\ell_{m}$, and whose the last intersection point between $\gamma$ and $\ell_{0}$ belongs to $\ell_{0}(x, y)$.

However only the inequality $K\left[\ell_{0}(0, L)\right] \geq K\left[\ell_{0}(0, L), \ell_{m}\right]$ holds a.s. (see Figure 8), by stationarity of the directed forest $\mathcal{F}_{-\hat{u}}$, it is possible to prove the identity

$$
\mathbb{E} K\left[\ell_{0}(0, L)\right]=\mathbb{E} K\left[\ell_{0}(0, L), \ell_{m}\right]
$$

for any integers $L, m>0$. See [5], Section 6, for details. Now, thanks to the coalescence of the semi-infinite geodesics of $\mathcal{F}_{-\hat{u}}$, the non-increasing sequence $\left(K\left[\ell_{0}(0, L), \ell_{m}\right]\right)_{m \geq 0}$ a.s. converges to a limit smaller than or equal to 1. By the Lebesgue's Dominated Convergence theorem, we get for any integer $L>0$ :

$$
L \mathbb{E} K\left[\ell_{0}(0,1)\right]=\mathbb{E} K\left[\ell_{0}(0, L)\right]=\lim _{m \rightarrow \infty} \mathbb{E} K\left[\ell_{0}(0, L), \ell_{m}\right] \leq 1 .
$$

This forces $\mathbb{E} K\left[\ell_{0}(0,1)\right]$ to be null. So, a.s. there is no bi-infinite geodesic crossing the vertical segment $\ell_{0}(0,1)$. We conclude by stationarity

Step 3: This third step consists in combining the results of the two previous ones in order to establish the convergence in probability of $\chi_{r}(\hat{u}, 2 \pi)$ to 0 . 


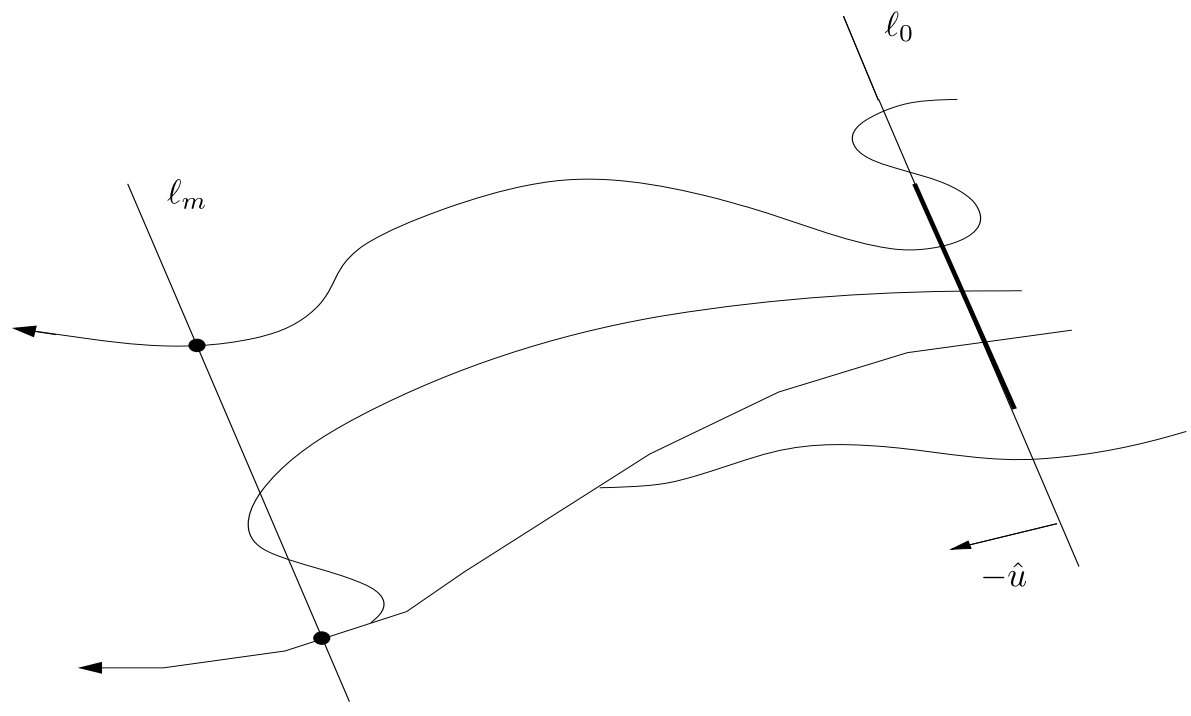

Figure 8. The thick segment on the line $\ell_{0}$ represents $\ell_{0}(x, y)$. The two black points on $\ell_{m}$ are elements counted by $K\left[\ell_{0}(x, y), \ell_{m}\right]$. On this picture, $K\left[\ell_{0}(x, y), \ell_{m}\right]$ equals 2 whereas $K\left[\ell_{0}(x, y)\right]$ equals 3 : two bi-infinite geodesics counted by $K\left[\ell_{0}(x, y)\right]$ merge before the line $\ell_{m}$.

Let $a(r)$ be the arc of the unit circle $S_{r}(o)$ centered at $r \hat{u}$ and with length $2 \pi$. When $r$ is large, $a(r)$ becomes close (for the Hausdorff distance) to the segment $I(r)$ centered at $r \hat{u}$ and with length $2 \pi$ which is orthogonal to $\hat{u}$. With high probability, the event $\chi_{r}(\hat{u}, 2 \pi) \geq 1$ implies the existence of a geodesic in the shortest-path tree $\mathcal{T}_{x^{\star}}$ crossing $I(r)$ and exiting the disk $B_{R}(r \hat{u})$, for any $R-$ when one walks away from the root $x^{\star}$. Precisely, for any $R>0$,

$$
\mathbb{P}\left(\chi_{r}(\hat{u}, 2 \pi) \geq 1\right) \leq P\left(U_{r}\right)+o(1),
$$

as $r$ tends to infinity, where the event $U_{r}$ is defined by: there exist $x_{1}, x_{2}, x_{3} \in X$ such that $\gamma_{x_{1}, x_{2}} \cap$ $I(r) \neq \varnothing, \gamma_{x_{1}, x_{2}} \subset B_{R}(r \hat{u}), \gamma_{x_{1}, x_{3}}=\gamma_{x_{1}, x_{2}} \cup\left[x_{2}, x_{3}\right]$ and $x_{3} \in B_{2 R}(r \hat{u}) \backslash B_{R}(r \hat{u})$. Inequality (7) relies on the fact that, with high probability when $R$ tends to infinity, the graph $G(X)$ contains no edge whose endpoints respectively belong to $B_{R}(r \hat{u})$ and the outside of $B_{2 R}(r \hat{u})$. In both cases $G(X)=\operatorname{Rng}(X)$ or $G(X)=\operatorname{Del}(X)$, this would imply the existence of a random disk avoiding the Poisson point process $X$, overlapping $B_{R}(r \hat{u})$ and with diameter larger than $R$. Let $\kappa=\lfloor 6 \pi\rfloor+1$. There exists a deterministic sequence $u_{1}, \ldots, u_{\kappa}$ of points of the circle $S_{3 R / 2}(r \hat{u})$ such that $\left|u_{i}-u_{i+1}\right| \leq R / 2$ for $i=1, \ldots, \kappa$ (where the index $i$ is taken modulo $\kappa$ ). Then, at least one of the deterministic disks $B_{R / 2}\left(u_{i}\right)$ would avoid the Poisson point process $X$. Such an event should not occur with high probability as $R$ tend to infinity (uniformly in $r$ ).

Since the geodesic $\gamma_{x_{1}, x_{3}}$ is included in $B_{2 R}(r \hat{u})$ then the event $U_{r}$ can be described using a local function with local parameter $2 R$. So, we can apply the result of Step 1 . Let $I(0)$ be the segment centered at the origin $o$ and with length $2 \pi$ which is orthogonal to $\hat{u}$. For any $R>0$, the probability $\mathbb{P}\left(\chi_{r}(\hat{u}, 2 \pi) \geq 1\right)$ is bounded by $\mathbb{P}\left(W_{r}\right)+o(1)$ as $r$ tends to infinity, where the event 
$W_{r}$ is defined by: there exists $x \in X \backslash B_{R}(o)$ whose semi-infinite geodesic $\gamma_{x}$ in $\mathcal{F}_{-\hat{u}}$ crosses the segment $I(0)$. Now, thanks to Step 2, there is no bi-infinite geodesic in the directed forest $\mathcal{F}_{-\hat{u}}$ with probability 1 . So we can choose the radius $R$ large enough so that $\mathbb{P}\left(W_{r}\right)$ is as small as we want.

Step 4: It then remains to state the following uniform moment condition to strengthen the convergence of $\chi_{r}(\hat{u}, 2 \pi)$ to 0 in the $L^{1}$ sense:

$$
\exists r_{0}>0, \quad \sup _{r \geq r_{0}} \mathbb{E} \chi_{r}(\hat{u}, 2 \pi)^{2}<\infty
$$

See [5], Section 3.1, for details. Let $\psi_{r}$ be the number of edges of the shortest-path tree $\mathcal{T}_{x^{\star}}$ crossing the arc $a(r)$. Of course, $\chi_{r}(\hat{u}, 2 \pi)$ is smaller than $\psi_{r}$ and it suffices - to get (8) - to prove that $\mathbb{P}\left(\psi_{r}>n\right)$ decreases fast enough and uniformly on $r$.

First, the number of Poisson points inside the disk $B_{R}(r \hat{u})$ is controlled by [18], Lemma 11.1.1: for any $n, r, R$,

$$
\mathbb{P}\left(\#\left(X \cap B_{R}(r \hat{u})\right)>n\right) \leq e^{-n \ln \left(\frac{n}{e \pi R^{2}}\right)} .
$$

[Note that (9) can also be obtained from Stirling's formula when $R$ and $n$ are large.] From now on, we assume that there are no more than $n$ Poisson points inside $B_{R}(r \hat{u})$. If the tree $\mathcal{T}_{x^{\star}}$ admits more than $n$ edges crossing the arc $a(r)$, that is, $\psi_{r}>n$, then necessarily one of these edges has (at least) one of its endpoints outside $B_{R}(r \hat{u})$. In both cases $G(X)=\operatorname{Rng}(X)$ or $G(X)=\operatorname{Del}(X)$, this implies the existence of a random disk avoiding the Poisson point process $X$, overlapping the arc $a(r)$ and with diameter larger than $R-2 \pi$. It is not difficult to prove that the probability of such an event decreases exponentially fast with $R$ and uniformly on $r$ and $\hat{u}$. That is to say there exist positive constants $c, c^{\prime}$ such that for $R$ large enough,

$$
\mathbb{P}\left(\#\left(X \cap B_{R}(r \hat{u})\right) \leq n, \psi_{r}>n\right) \leq c e^{-c^{\prime} R} .
$$

The searched result follows from (9) and (10) by taking for example $R=n^{1 / 4}$.

\section{Acknowledgements}

The authors thank the anonymous referees for their detailed comments and corrections on a previous version of the manuscript. Their reports helped us to improve substantially the quality of the present article. The major part of this work was done when C. Hirsch was a postdoc at the Weierstrass Institute.

D. Coupier was supported by the CNRS GdR 3477 GeoSto, the Labex CEMPI (ANR-11LABX-0007-01) and the P4 project (ANR-16-CE40-0016). C. Hirsch was supported by the Leibniz program Probabilistic Methods for Mobile Ad-Hoc Networks. This research publication was funded by LMU Munich's Institutional Strategy LMUexcellent within the framework of the German Excellence Initiative. 


\section{References}

[1] Aldous, D.J. (2009). Which connected spatial networks on random points have linear route-lengths? Preprint. Available at arXiv:0911.5296.

[2] Baccelli, F., Coupier, D. and Tran, V.C. (2013). Semi-infinite paths of the two-dimensional radial spanning tree. Adv. in Appl. Probab. 45 895-916. MR3161288

[3] Baccelli, F., Tchoumatchenko, K. and Zuyev, S. (2000). Markov paths on the Poisson-Delaunay graph with applications to routing in mobile networks. Adv. in Appl. Probab. 32 1-18. MR1765174

[4] Chenavier, N. and Devillers, O. (2015). Stretch factor of long paths in a planar Poisson-Delaunay triangulation. Preprint.

[5] Coupier, D. (2016). Sublinearity of the number of semi-infinite branches for geometric random trees. Preprint. Available at arXiv:1501.04804.

[6] Coupier, D. and Tran, V.C. (2013). The 2D-directed spanning forest is almost surely a tree. Random Structures Algorithms 42 59-72. MR2999212

[7] Daley, D.J. and Last, G. (2005). Descending chains, the lilypond model, and mutual-nearest-neighbour matching. Adv. in Appl. Probab. 37 604-628. MR2156551

[8] Dobkin, D.P., Friedman, S.J. and Supowit, K.J. (1990). Delaunay graphs are almost as good as complete graphs. Discrete Comput. Geom. 5 399-407. MR1043722

[9] Hammersley, J.M. and Welsh, D.J.A. (1965). First-passage percolation, subadditive processes, stochastic networks, and generalized renewal theory. In Proc. Internat. Res. Semin., Statist. Lab., Univ. California, Berkeley, Calif. 61-110. New York: Springer. MR0198576

[10] Hirsch, C., Neuhäuser, D. and Schmidt, V. (2016). Moderate deviations for shortest-path lengths on random segment processes. ESAIM Probab. Stat. 20 261-292. MR3528627

[11] Howard, C.D. and Newman, C.M. (1997). Euclidean models of first-passage percolation. Probab. Theory Related Fields 108 153-170. MR1452554

[12] Howard, C.D. and Newman, C.M. (2001). Geodesics and spanning trees for Euclidean first-passage percolation. Ann. Probab. 29 577-623. MR1849171

[13] Jaromczyk, J.W. and Toussaint, G.T. (1992). Relative neighborhood graphs and their relatives. Proc. IEEE 80 1502-1517.

[14] Keil, J.M. and Gutwin, C.A. (1992). Classes of graphs which approximate the complete Euclidean graph. Discrete Comput. Geom. 7 13-28. MR1134449

[15] Licea, C. and Newman, C.M. (1996). Geodesics in two-dimensional first-passage percolation. Ann. Probab. 24 399-410. MR1387641

[16] Newman, C.M. (1995). A surface view of first-passage percolation. In Proceedings of the International Congress of Mathematicians, Vol. 1, 2 (Zürich, 1994) 1017-1023. Basel: Birkhäuser. MR1404001

[17] Pimentel, L.P.R. (2011). Asymptotics for first-passage times on Delaunay triangulations. Combin. Probab. Comput. 20 435-453. MR2784636

[18] Talagrand, M. (1995). Concentration of measure and isoperimetric inequalities in product spaces. Inst. Hautes Études Sci. Publ. Math. 81 73-205. MR1361756

Received October 2016 and revised March 2017 